\title{
Recent progresses of quantum confinement in graphene quantum dots
}

\author{
Si-Yu Li ${ }^{1, \dagger}$, Lin $\mathbf{H e}^{2, \ddagger}$ \\ ${ }^{1}$ Key Laboratory for Micro-Nano Physics and Technology of Hunan Province, College of Materials Science \\ and Engineering, Hunan University, Changsha 410082, China \\ ${ }^{2}$ Center for Advanced Quantum Studies, Department of Physics, Beijing Normal University, Beijing 100875, China \\ Corresponding authors. E-mail: ${ }^{\dagger}$ lisiyu@hnu.edu.cn, ${ }^{\ddagger}$ helin@bnu.edu.cn \\ Received September 9, 2021; accepted October 18, 2021
}

Graphene quantum dots (GQDs) not only have potential applications on spin qubit, but also serve as essential platforms to study the fundamental properties of Dirac fermions, such as Klein tunneling and Berry phase. By now, the study of quantum confinement in GQDs still attract much attention in condensed matter physics. In this article, we review the experimental progresses on quantum confinement in GQDs mainly by using scanning tunneling microscopy (STM) and scanning tunneling spectroscopy (STS). Here, the GQDs are divided into Klein GQDs, bound-state GQDs and edge-terminated GQDs according to their different confinement strength. Based on the realization of quasi-bound states in Klein GQDs, external perpendicular magnetic field is utilized as a manipulation approach to trigger and control the novel properties by tuning Berry phase and electron-electron $(\mathrm{e}-\mathrm{e})$ interaction. The tip-induced edge-free GQDs can serve as an intuitive mean to explore the broken symmetry states at nanoscale and single-electron accuracy, which are expected to be used in studying physical properties of different two-dimensional materials. Moreover, high-spin magnetic ground states are successfully introduced in edge-terminated GQDs by designing and synthesizing triangulene zigzag nanographenes.

Keywords graphene quantum dot, scanning tunneling microscopy, scanning tunneling spectroscopy, quasi-bound state, bound state, triangulene

Contents

1 Introduction

2 Quasi-bound states in Klein GQDs

2.1 Imaging "whispering galley mode" in Klein GQDs

2.2 Novel phenomena induced by magnetic field in Klein GQDs

2.2.1 On/off Berry phase switch in circular Klein GQDs

2.2.2 Quantum Hall wedding cake-like structures in Klein GQDs

2.3 Coulomb interaction-induced splitting of quasi-bound states

2.4 Relativistic artificial molecules realized by two coupled Klein GQDs

3 Bound states in GQDs

3.1 GQDs in continuous graphene sheet via strong coupling of substrate

3.2 STM tip-induced edge-free GQDs

3.3 Novel bound states in bilayer GQDs

\footnotetext{
* This article can also be found at http://journal.hep.com. $\mathrm{cn} /$ fop/EN/10.1007/s11467-021-1125-2.
}

4 Edge-terminated confinement in triangular

1

7 Quantum dots (QDs) could be applied to form spin qubit, which initially arouses the interest of many scientists [1].

8 At around 2004, semiconductor QDs based on GaAs tech8 nology have been realized [2-5], however, the spin decoherence effect greatly limits their further application comes an excellent candidate for spin qubit, because of its weak spin-orbital coupling and weak hyperfine interaction, which effectively weaken the spin decoherence effect [6]. Therefore, graphene quantum dots (GQDs) have attracted much attention over the years. Besides of the potential application on spin qubit, plenty of experimental and theoretical works have found that GQDs provide 
an essential platform to study the fundamental properties of Dirac fermions, such as Klein tunneling [7-9], quantum electron optics [10,11], Berry phase [12, 13], and electronelectron (e-e) interaction [14]. By now, the study of quantum confinement in GQDs is still one of the hottest topics in condensed matter physics.

Researchers have developed several different methods to realize quantum confinement in graphene with different confinement strengths $[7,8,10,15-21]$, as schematically shown in Fig. 1(a). First, electrostatic potential is a preferred method to confine electrons in graphene (the weak confinement of Dirac Fermions in graphene). Since Klein tunneling makes electrostatic potentials become transparent to massless Dirac fermions (100\% transmission) at normal incidence, researchers choose to build circular graphene $\mathrm{p}-\mathrm{n}$ junctions to localize Dirac Fermions and form quasi-bound states $[7,8,10,15]$ [as shown in Fig. 1(a)]. When applying external perpendicular magnetic fields, the quasi-bound states show more novel phenomenon related to Berry phase [12], e-e interaction [14] and so on. The quasi-bound states and their correlated states at external magnetic fields in graphene $\mathrm{p}-\mathrm{n}$ junctions will be introduced in Section 2. Second, combining electrostatic potentials and energy gaps could realize bound states in GQDs [Fig. 1(b)], which exhibit singleelectron charging effects $[16,17,22-24]$. The singleelectron effect is always treated as a signal of that the GQDs are isolated with the surrounding regions by tunnel barriers. Surprisingly, the quadruplets of charging peaks resulting from bound states in tip-induced GQDs could be applied to study the degeneracy and broken symmetry states of graphene systems $[13,17,23]$. We will introduce the bound states in GQDs in Section 3. Thirdly, the strongest confinement in graphene could be realized by forming edge-terminated nanographenes, such as tri- angulene and its dimer as shown in Fig. 1(c) [19-21]. The characterization and unconventional magnetism of edgeterminated GQDs will be discussed in Section 4.2. Finally, in Section 5, we summarize the review and present outlook in this field.

\section{Quasi-bound states in Klein GQDs}

The tunneling of Dirac fermions across potential barriers in graphene obeys the so-called "Klein paradox", in which the incoming Dirac electrons at normal incidence will penetrate high and wide potential barriers with the transmittance of $100 \%$ [25-31]. Klein tunneling in graphene arises from its unusual gapless band structure and honeycomb lattice with sublattice isospin degree of freedom [26]. However, when crossing potential barriers at oblique incidence, both reflection and transition occur with reflection becoming dominate at large oblique angles, which makes it possible to trap electrons via forming circular graphene $\mathrm{p}-\mathrm{n}$ junction [32-39]. As shown in Fig. 1(a), the massless Dirac Fermions incident at large angles will reflect with high possibility at the boundary of circular graphene $n-p-n$ junction, and may reflect many times back to the original place. The reflected and incident electrons interfere with each other and form the quasi-bound states before they finally escape from the circular graphene $n-p-n$ junction [10]. Here, the circular graphene $n-p-n$ junctions with confined quasi-bound states are called Klein GQDs [40]. The quasi-bound states in the Klein GQDs are quite similar to the whispering galley mode (WGM) in microcavity, where the interference between the reflected and incidence waves results in the formation of standing waves $[10,11,15]$. In the Klein GQDs, electrons behave like acoustic waves in the microcavity. Because massless (a)

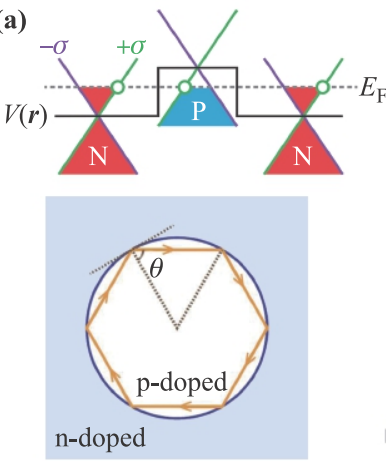

Weak confinement (b)

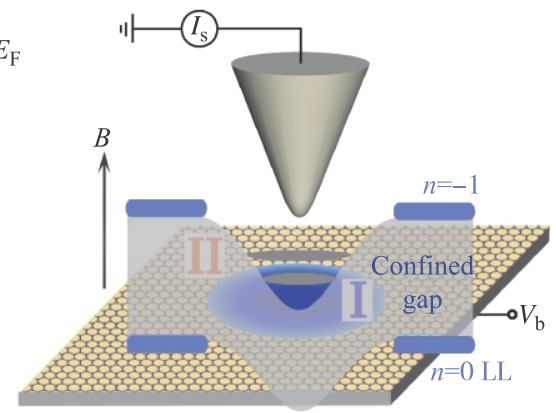

(c)
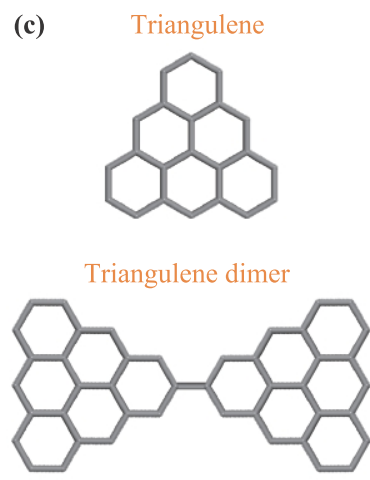

Strong confinement

Fig. 1 (a) Upper panel: Schematic of Klein tunneling across a graphene $n-p-n$ junction [8]. Lower panel: Schematic of a circle GQD with a typical closed interference path of the trapping Dirac fermions [10]. (b) Schematic of the tip-induced edge-free GQD with bound states. The tip-induced electrostatic potential results in LL bending in the region I beneath the STM tip. And the gaps between the graphene LLs provide the confined gaps. (c) Schematic of a triangulene and a triangulene dimer via bottom-up synthesizing method. 
Dirac fermions have the possibility to escape from the Klein GQDs, the quasi-bound states are quite different from the bound states in previous QDs. In this section, we will expound the characteristics of quasi-bound states in the Klein GQDs, and discuss their corresponding novel phenomena in magnetic fields.

\subsection{Imaging "whispering galley mode" in Klein GQDs}

In recent years, many research groups have acquired the Klein GQDs and spatially imaged the wavefunctions of the quasi-bound states with scanning tunneling microscopy (STM) $[7,8,10-12,14,15]$. There are mainly three different methods to realize Klein GQDs with sizes ranging from hundreds of nanometers to several nanometers.

First, using the electric field between STM tip and sample could form circular $\mathrm{p}-\mathrm{n}$ junctions beneath the tip, and STM tip acting as a top gate to tuning the poten- tial barriers in the meanwhile $[11,15]$. However, since the tip-induced potential barrier moves with the STM tip, it is different to image the wavefunctions of quasibound states. Second, researchers apply a voltage pulse via STM tip to the graphene layer placing on top of hexagonal boron nitride $(\mathrm{hBN})$. The tip pulse-induced electric field ionizes the impurities in the $\mathrm{hBN}$ region beneath the STM tip, then forms a stationary screening charge distribution area on the $\mathrm{hBN}$ substrate $[7,12]$. Therefore, a fixed circular graphene $\mathrm{p}-\mathrm{n}$ junction is realized. We could detect the electronic structures at different locations inside and outside the dots, and directly image the whispering galley modes in real space. Large-sized Klein GQDs with radius over one hundred nanometers are always created using the two methods above, because the potential of the STM tip or voltage pulsing usually have large-scale influence beneath the tip. Scientists also find another method to generate nano-scale Klein GQDs on $\mathrm{Cu}$ (a)

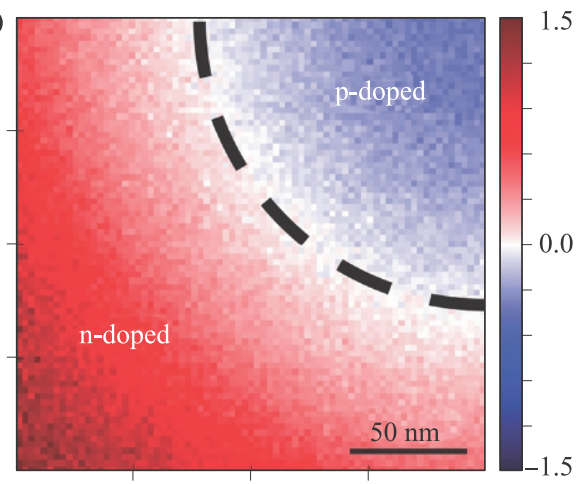

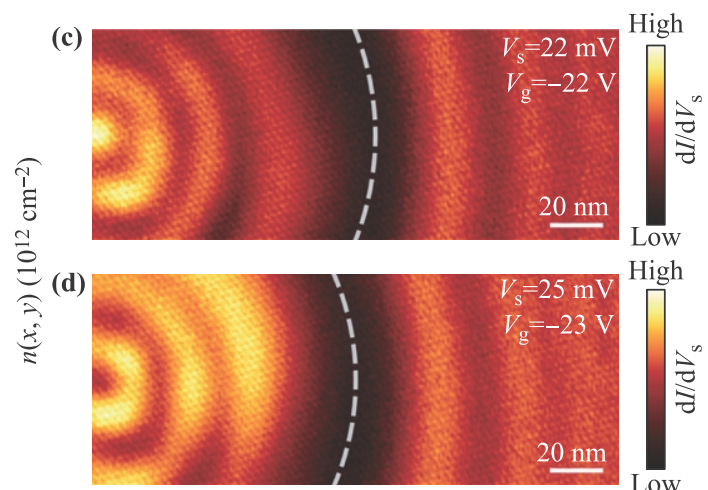

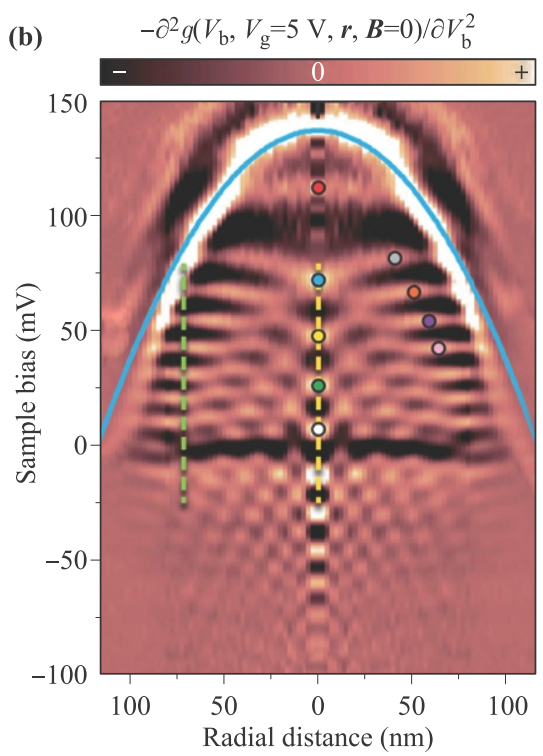

(e)

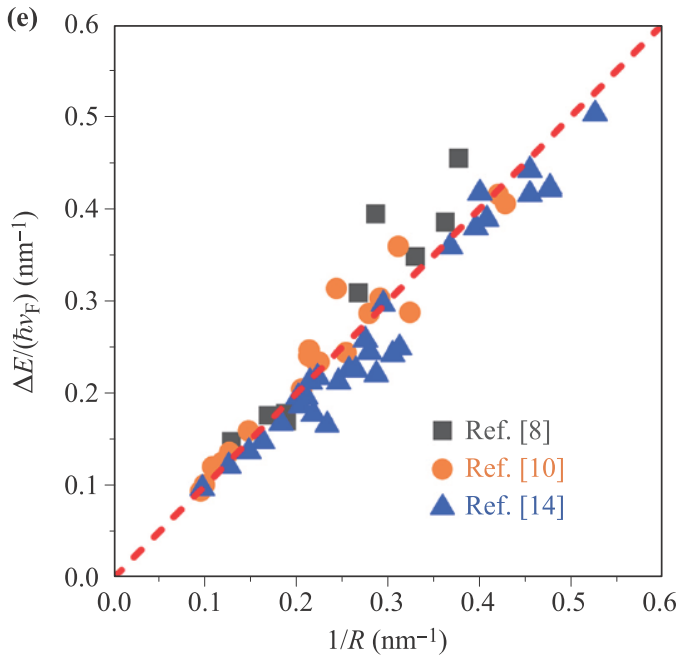

Fig. 2 (a) The charge density map for one quadrant of a large-scale Klein GQD [7]. (b) STS spectra map versus radial spatial position obtained across a circular Klein GQD. The yellow dashed line marks the $m= \pm 1 / 2$ states appearing in the center [12]. (c), (d) STS maps of a large-scale Klein GQD at different energies, which image the quantum interferences inside and outside the $\mathrm{p}-\mathrm{n}$ junction [7]. (e) Summarizing the energy spacings of quasi-bound states as function of the inverse radium $1 / R$ in different nanoscale Klein GQDs. 
substrate via chemical vapor deposition (CVD) progress. During the synthesis progress of graphene on $\mathrm{Cu}$ foil, some vacancy islands on $\mathrm{Cu}$ surface are formed with sizes ranging from several nanometers to scores of nanometers, because of the migration and diffusion of the surface $\mathrm{Cu}$ atoms at high-temperature annealing process. For the as-grown graphene layers on $\mathrm{Cu}$ substrate, the space between graphene and the location of $\mathrm{Cu}$ vacancy island is larger than that in other $\mathrm{Cu}$ surface regions, leading to a sharp electronic junction, thus forming a nanoscale Klein GQD $[8,10,14]$.

Figure 2(a) shows the charge density map of a typical fixed Klein GQDs with radius about $100 \mathrm{~nm}$ realized via the second method [7]. The potential inside the well of $n-$ $\mathrm{p}-\mathrm{n}$ junction is higher than the outside, thus such a well provides a trapping potential to holes, not for electrons. Seeing from the STS spectra map in Fig. 2(b), confining holes inside the Klein GQD forms a series of quasibound states exiting within an envelope region marked by the blue solid line which corresponds to the parabolic potential inside the confining circular $\mathrm{p}-\mathrm{n}$ junction [12]. The energy separation between the adjacent quasi-bound states obviously decreases with the detected position moving away from the dot center. Because of the rotational symmetry, it is useful to describe quasi-bound states in circular resonator by radial quantum numbers $n$ and angular momentum quantum numbers $m$. Only the $m= \pm 1 / 2$ quasi-bound states with different $n$ values appear in the center region of the Klein GQDs. The states with higher angular momentum states are distributed in the region away from the resonator's center, because that electrons with higher angular momentum are repelled from the center by the centrifugal barrier [7, 12, 15]. From the STS maps in Figs. 2(c) and (d), we can directly image the eigenstate distributions of the quasi-bound states at different energies in a circular Klein GQD [7]. The node/antinode annular patterns inside the Klein GQD in Fig. 2(c) are quite different from that in Fig. 2(d), one has a bright node at the center, whereas the other presents anti-nodes at the center, which correspond to the quasi-bound states with $m= \pm 1 / 2$ and another $m$ value respectively. The interference patterns outside the Klein GQD in Figs. 2(c) and (d) are attributed to Friedel oscillations induced by scattering of quasiparticles at the barrier boundary [7].

Different from the results of the above large-scale Klein GQDs, the observed resonance peaks of quasi-bound states are almost equally spaced at different positions inside the nanoscale Klein GQDs $[8,10,14]$. The tiny size of nanoscale Klein GQDs makes them hard to trap the quasi-particles with high energies, therefore, only lowestlying resonant states for small angular momentum are detected and they exhibit almost equal-spaced in a size-fixed nanoscale Klein GQDs. According to the theoretical calculation for Dirac electrons confined in a nanoscale circular $\mathrm{p}-\mathrm{n}$ junction with the radium $R$, these lowest-lying resonant states are almost spaced linearly following the relationship of $\Delta E \approx \alpha \hbar \nu_{\mathrm{F}} / R$, where $\nu_{\mathrm{F}} \approx 1 \times 10^{6} \mathrm{~m} / \mathrm{s}$ is Fermi velocity, $\hbar$ is the reduced Planck constant, and $\alpha$ is the dimensionless constant of order unity $[8,10,14]$. We summarize the energy spacings of quasi-bound states as function of the inverse radium $1 / R$ of different nanoscale Klein GQDs reported in literatures in Fig. 2(e), which exhibits a linear relationship and yields the value of $\alpha \approx 1$ from the linear fitting $[8,10,14]$. Based on the modulation of vacancy islands on $\mathrm{Cu}$ surface via the third method, the Klein GQDs could be formed with different sizes and geometries. For example, the quasi-rectangular Klein GQD is obtained which exhibits much more complex spatial distributions of LDOS for quasi-bound states inside the dot [10]. The realization of the Klein GQDs with different sizes and geometries makes it possible to manipulate refraction and transmission of Dirac fermions and explore quantum electron optics in graphene.

\subsection{Novel phenomena induced by magnetic field in Klein GQDs}

When magnetic field is added, the combination of spatial confinement by electric field and magnetic confinement by cyclotron motion will result in novel phenomena in Klein GQDs $[12,41,42]$. In weak magnetic field, the degenerate $\pm m$ quasi-bound states exhibit large splittings, because the trajectories of charge carriers are bended into a "skipping" obit with loops and a $\pi$ Berry phase is switched on when a small critical magnetic field is reached [12, 43] [Figs. 3(a), (b)]. At larger magnetic fields, the Klein GQD systems enter the quantum Hall regime, with the quasibound states condensing into highly degenerate Landau levels (LLs) [Figs. 3(b), (c)]. The corresponding screened potential turns into a wedding cake-like structure induced by e-e interaction [44-48] [Fig. 3(d)]. The phenomena of on/off Berry phase switch and interaction-driven quantum Hall wedding cake-like structures are directly observed in the Klein GQDs via STM measurements as described below $[12,42]$.

\subsubsection{On/off Berry phase switch in circular Klein GQDs}

Berry phase is the accumulated geomatic phase when a state evolves adiabatically along a circle in momentum space according to Schrödinger's equation [49]. The novel topological properties in many quantum systems are always attributed to their nontrivial Berry phase $[13,49$ 56]. In monolayer graphene, the Berry phase can take only two values, 0 or $\pi$, because of the spin-momentum locking. The pseudospin $1 / 2$ of massless fermions rotates by $2 \pi$ along a closed Fermi surface results in the Berry phase $\pi$ of monolayer graphene, which is responsible for the Klein tunneling [30, 31] and the unconventional "half-integer" quantum Hall effect [56-58]. The value of Berry phase always stays static and is hard to be controlled because controlling the trajectories of Dirac fermions in graphene is 
(a)

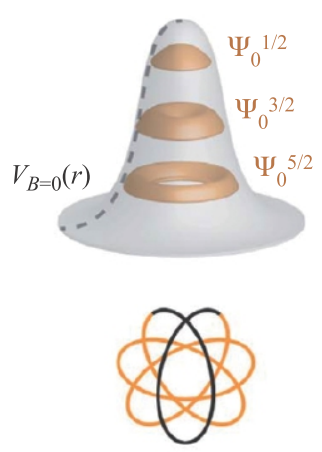

Low magnetic field (b)

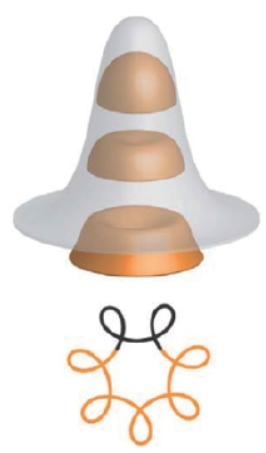

(c)

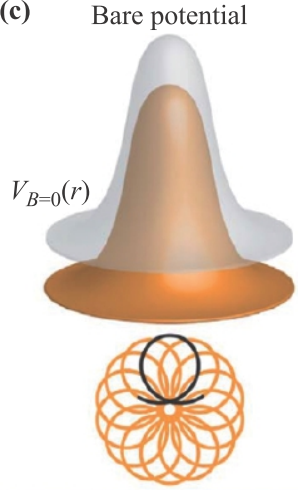

(d) Screened potential

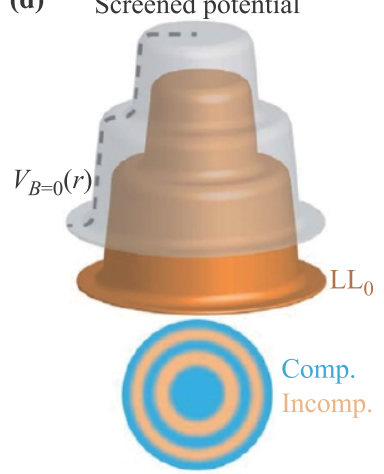

High magnetic field

Fig. 3 ( $\mathbf{a}-\mathbf{d})$ Upper panels: Schematic grey surfaces correspond to potential profiles and the orange surfaces correspond to the wave function density with increasing magnetic field. Lower panels: The corresponding semiclassical orbits of charge carriers in the Klein GQDs with increasing magnetic field [42].

experimentally challenging. The Klein GQDs $[7,8,10,15]$ makes it possible to exquisitely control the electron orbits by forming quasi-bound states, and provide an excellent platform to alter and measure the Berry phase of electron orbital states [12].

Figure 3(a) schematically shows the charge trajectories for positive $m$ states inside the circular n-p-n Klein GQD. External magnetic field will bend the trajectories of charge carriers by Lorentz force and change the incident angle at p-n boundary inside the Klein GQDs. When a small critical magnetic field $B_{c}$ reached, the orbit with angular momentum antiparallel to the magnetic field is bended into a "skipping" orbit with loops [Fig. 3(b)] [12, 59]. During this transition, the trajectory in momentum-space is changed to enclosing the Dirac point at critical magnetic field, and the value of Berry phase discontinuously jumps from 0 towards $\pi$. The sudden change of Berry phase results in the shifting of energy levels accordingly: the $\pm m$ states in the Klein GQDs, which are degenerate at $B=0$, are separated apart by half a period at the critical magnetic field [12]. Figure 4(a) shows the magnetic field dependence of the $m= \pm 1 / 2$ states for the modes from $n=1$ to $n=5$ measured at the center of a fixed Klein GQD. With increasing the magnetic field, new resonator states suddenly appear between the $n$th quasi-bound states at $B_{c} \approx 0.11 \mathrm{~T}$. The separation of the new states $\delta \varepsilon_{m}$ at magnetic fields, which is on the order of $10 \mathrm{meV}$, is about a half of the separations between the $n$th quasi-bound states. The new resonator states are resulted from the energy shifts of the $n$th quasi-bound states when a $\pi$ Berry phase is switched on at a small critical magnetic field. The magnified view of $n=4, m= \pm 1 / 2$ modes in Fig. 4(b) shows the Berry phase-induced jumps of $m= \pm 1 / 2$ states in magnetic fields: the $m=1 / 2$ state jumps at positive critical magnetic fields, and the $m=-1 / 2$ state jumps at negative critical magnetic fields, which finally leads to the observed "new states" between $n$th quasi-bound states.
The summary of the separation $\delta \varepsilon_{m}$ versus magnetic fields in Fig. 4(c) directly illustrates the switching of the Berry phase.

On/off Berry phase switching cannot be realized by purely using magnetic field or electrostatic potentials. The realization of controlling a $\pi$ Berry phase relies on the confinement of Dirac fermions in Klein GQDs, which extends the possible application of the Klein GQDs on graphene-based electron optics.

\subsubsection{Quantum Hall wedding cake-like structures in Klein GQDs}

The above on/off Berry phase switch in the Klein GQDs is realized by small magnetic field [12] [Figs. 3(a), (b)]. With increasing magnetic field, the combination of the spatial confinement by electric field and the magnetic confinement will strengthen the effect of e-e interaction in the Klein GQDs [42]. At large magnetic fields, the Klein GQD enters the quantum Hall regime, with the quasi-bound states [Figs. 3(a), (b)] condensing into highly degenerate Landau levels (LLs) [Fig. 3(c)]. Then, screened potential is changed into a wedding cake-like appearance by e-e interaction [Fig. 3(d)]. Therefore, there will be wedding cakelike structures formed in the electron density consisting of a series of compressible and incompressible electron liquid rings [44-48], as schematically shown in the lower panel of Fig. 3(d). Such features could be directly mapped spatially in STM measurement.

When the magnetic length becomes smaller than the confining potential width, the transition from spatial confinement to magnetic confinement will occurs. At zero field, the screened confining potential $V_{B=0}(r)$ in a Klein GQD could be modeled by

$$
V_{B=0}(r) \approx U_{0} \exp \left(-\frac{r^{2}}{R_{0}^{2}}\right)+U_{\infty}
$$



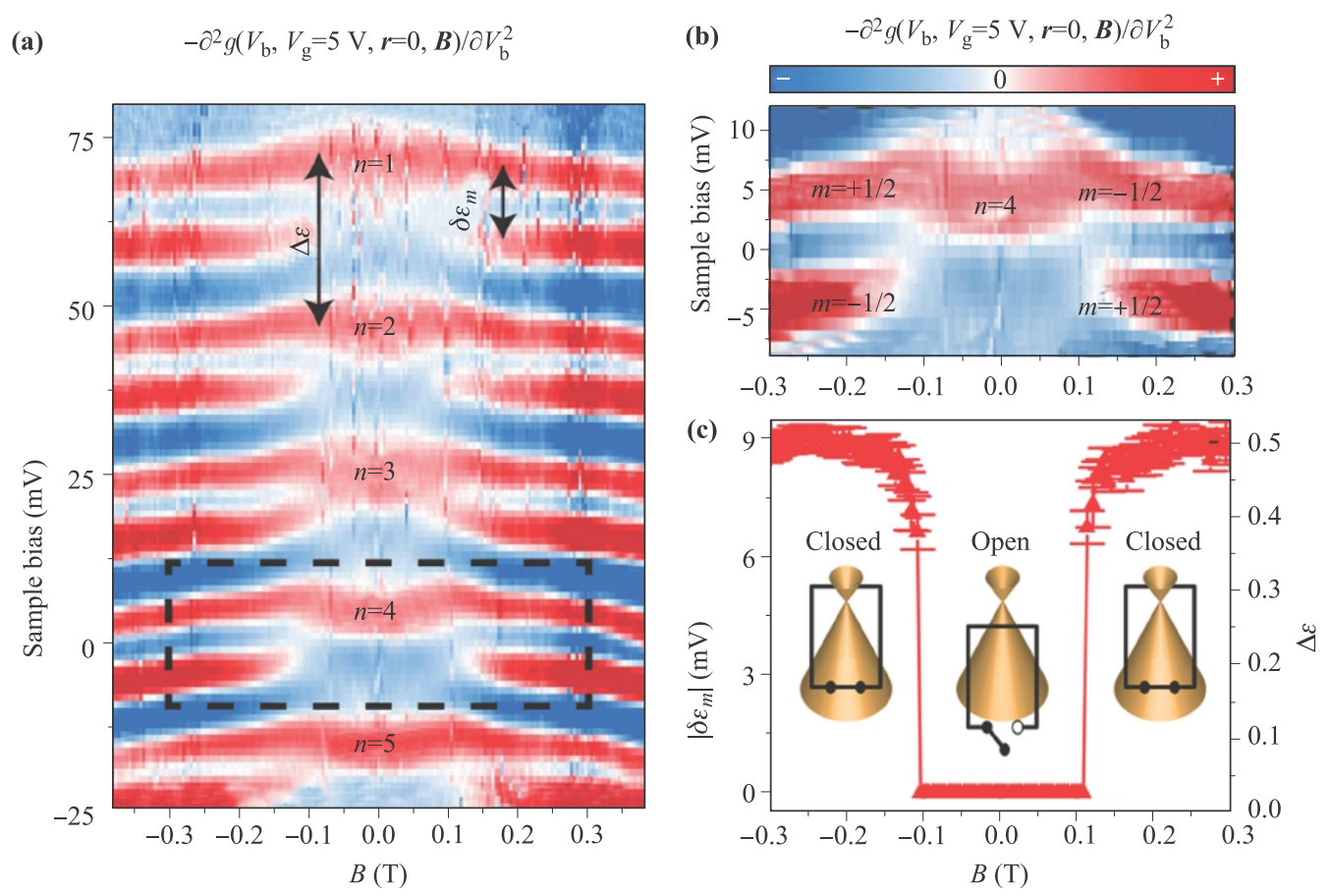

Fig. 4 (a) STS map versus magnetic field taken in the center of the circular Klein GQD with $n=1$ to $n=5$ modes. New resonator states appear at critical magnetic field $B_{c} \approx \pm 0.11 \mathrm{~T}$. (b) Magnified $n=4$ modes in panel (c) to show the jumps of $m= \pm 1 / 2$ states versus magnetic field. (c) The energy difference between $m=1 / 2$ state and $m=-1 / 2$ state, $\delta \varepsilon_{m}$, for $n=4$ mode various with magnetic field. The schematic Dirac cones show the switching action: for low magnetic fields $B<B_{c}$, the switch is open with zero Berry phase; when $B_{c}$ is reached, the switch is immediately closed and a $\pi$ Berry phase is turned on $[12]$.

where $U_{0}$ is electric potential at the center of the Klein GQD, $R_{0}$ is the diameter of the dot, $U_{\infty}$ is the background value, and $r$ is the distance from the center of the GQD $[12,42]$. At high magnetic field incorporating e-e interaction, the effective potential $V_{B}$ including the effect of screening is reduced compared with $V_{B=0}(r)$ :

$$
V_{B}(r)=V_{e x t}(r)+\int \mathrm{d}^{2} r^{\prime} V_{e e}\left(\left|r-r^{\prime}\right|\right) n\left(r^{\prime}\right),
$$

where $n(r)$ is the charge density at position $r, V_{\text {ext }}$ is the electrostatic potential of the dot, $V_{e e}(r)$ is the Coulomb interaction $[42,44]$. Figures 5(a) and (b) show the simulated effective potential $V_{B}$ and charge density $n(r)$ of the Klein GQD at different magnetic fields [42]. The joint effect in magnetic field and e-e interaction create a series of plateaus, resulting in the formation of wedding cakelike patterns of concentric rings inside the dots. In order to reflect the effect of interaction on LLs inside the Klein GQDs, they compare the LDOS calculated with and without e-e interaction in Fig. 5(c) [42]. Without e-e interaction [the right panel of Fig. 5(c)], the LDOSs of LLs inside the dot follow the tendency of the potential $V_{B=0}(r)$. After considering the e e interaction, the evolution of LLs LDOS follows that of the interaction-induced $V_{B}(r)$, where the LLs flatten in the center and shift to- wards the lower energies.

By taking STM measurement, the wedding cake-like structures are directly observed in the LDOS map at $4 \mathrm{~T}$ versus distance within the Klein GQD, as shown in Fig. 5(d) [42]. Here, many quasi-bound states disappear and condense into degenerated LLs. The $N=-5$ to $2 \mathrm{LLs}$ are observed, exhibiting as plateaus in the center of the dot, and the $N=0,+1$ LLs develop into the additional kinks near the QD boundary. The extra concave features in the effective potential in the center region are also observed in the map of LLs LDOS, which are accorded with the theoretical results. The spatial STS map at Fermi level at $4 \mathrm{~T}$ in Fig. 5(e) shows a $\mathrm{LL}(-1)$ compressible disk in the center and a $\operatorname{LL}(0)$ compressible ring near the dot boundary separated by an incompressible ring. This result further confirms the theoretical prediction in Fig. 3(d). When LLs cross the Fermi level, the Coulomb charging effect starts to appear, as indicated by the quartet rings in the center and out of the dot edge [Fig. 5(e)] [24, 42].

The wedding cake-like patterns in the Klein GQDs is a clear and notable signature of the e-e interaction. In their experiment, another unexpected feature is the appearance of circle nodal patterns in the spatial STS maps even at zero magnetic field, which may be arising from Wigner crystals-like effect induced by interactions [42, 47, 60-62]. 
(a)

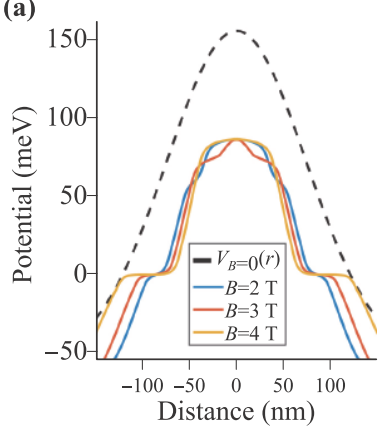

(b)

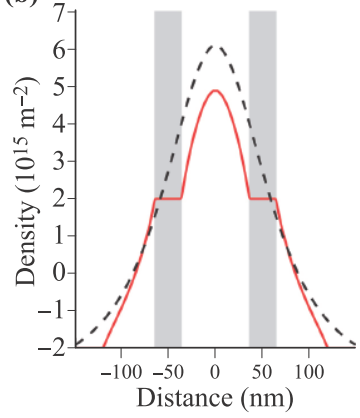

(c)

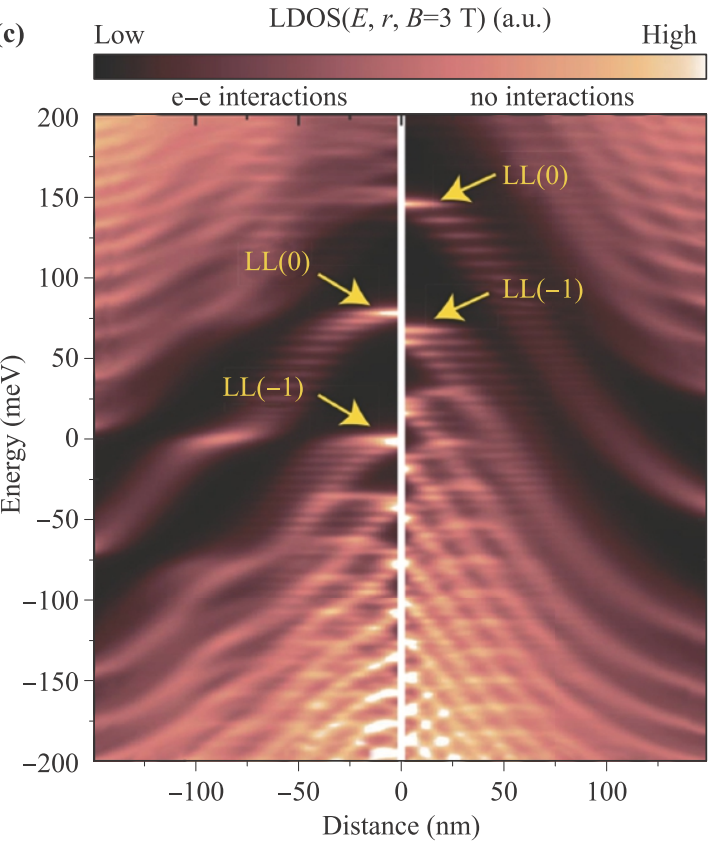

(d)
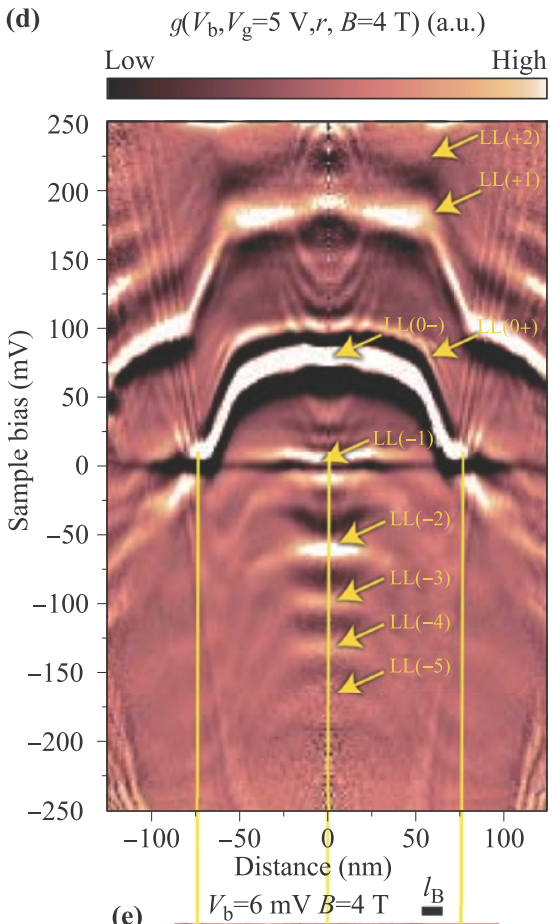

(e)

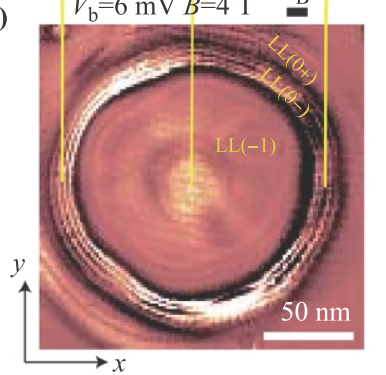

Fig. 5 (a) Simulated effective potential according to Eq. (2) at several different magnetic fields (solid lines), and the $V_{B=0}(r)$ curve marked by a dashed line in Klein GQD. (b) The simulated carrier density considering e-e interaction at 4 T in Klein GQD. (c) The simulated LDOS map using the potential at $B=3 \mathrm{~T}$ and $V_{B=0}(r)$ from panel (a), respectively. (d) Experimental STS map as function of distance $r$ and $V_{\mathrm{b}}$ at $4 \mathrm{~T}$, exhibiting the wedding cake-like structures in the LLs inside the Klein GQD. (e) Spatial STS maps of the Klein GQD in $4 \mathrm{~T}$ taken at $V_{\mathrm{b}}=6 \mathrm{mV}$ (near Fermi level) [42].

\subsection{Coulomb interaction-induced splitting of quasi-bound states}

Coulomb interaction mainly manifests itself at Fermi level when the flat bands or the bound states are partially filled [62-82]. The Coulomb interaction-induced splitting of flat bands and bound states at partially filling have been observed in previous studies [62-82]. Recently, it has been demonstrated that the Coulomb interaction could also split the partially filled quasi-bound states in Klein GQDs [14], even though the trapping time of the quasibound states is only in the range of $10 \mathrm{fs}[8,10]$. In the STS measurements, the partially filled quasi-bound state splits into two peaks, while the other fully-occupied or emptyoccupied quasi-bound states in a Klein GQD do not show splitting. Otherwise, for the same quasi-bound state, it shows obvious splitting at partially filling, and exhibits no signal of splitting no matter it is fully-occupied or emptyoccupied. The splitting of the partially filled quasi-bound states in Klein GQDs is attributed to Coulomb interaction at Fermi level [14]. The splitting of partially filled quasi-bound states are different in different Klein GQD with various radii $R$, as shown in Figs. 6(a) and (b). It is interesting to find that the splitting increases linearly with $1 / R$, which could be well decribed by the equation of on-site Coulomb repulsion:

$$
U=\frac{1}{4 \pi \varepsilon} \frac{e^{2}}{R} .
$$

Here, $\varepsilon$ is the effective dielectric constant of the dot, and $e$ is the electron charge [83]. Through the linear fitting according to Eq. (3) in Fig. 6(b), the effective dielectric constant of the dot is yielded as $\varepsilon \approx(6.62 \pm 0.25) \varepsilon_{0}$. Here 


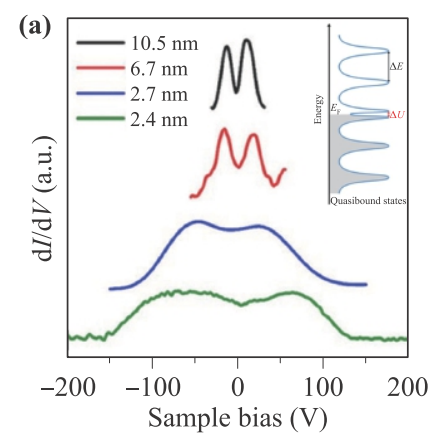

(c)

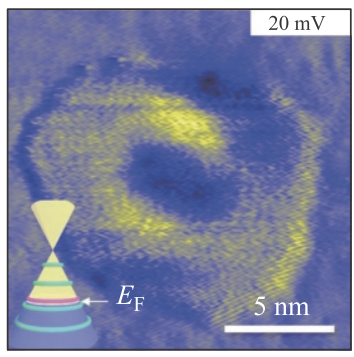

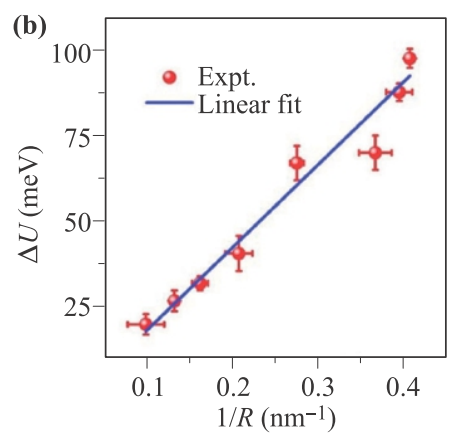

(d)

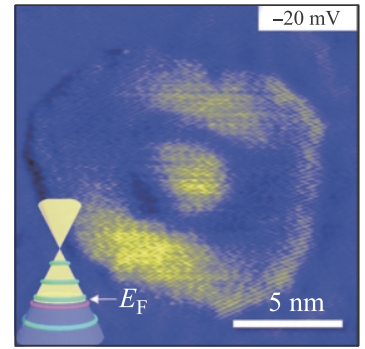

Fig. 6 Four normalized $\mathrm{d} I / \mathrm{d} V$ spectra with the splitted quasi-bound states taken at four different Klein GQDs with different radii. Inset: Schematic of filling-related splitting of quasi-bound states. (b) Summarize the splitting energies of partially filled quasi-bound states in different Klein GQDs as a function of the inverse effective radii $1 / R$. The blue solid line marks the linear fit according to Eq. (3). (c, d) $\mathrm{d} I / \mathrm{d} V$ mappings taken at the energies of the two split peaks of the partially filled quasi-bound state, where the WGM is broken [14].

$\varepsilon_{0}$ is the vaccuum dielectric constant [84-88]. On the other hand, the Coulom interaction also breaks the corresponding WGM of the partially filled quasi-bound states [14]. For the splitted quasi-bound states induced by Coulomb interaction at partially filling, the LDOS of the occupied state (left peak) and the LDOS of the empty state (right peak) distribute separately at different regions of the Klein GQD, as shown in Figs. 6(c) and (d). Such a distribution effectively reduces the Coulomb interaction and finally results in the broken of the WGM.

The Coulomb interaction not only splits the partially filled quasi-bound states, but also breaks their corrsponding WGMs in the Klein GQDs. However, it is still unclear the nature of the Coulomb-induced splitting of the quasi-bound states and further work needs to carry out to explore it.

\subsection{Relativistic artificial molecules realized by two coupled Klein GQDs}

Two coupled QDs are always treated as an artifical molecule [89-95] with coherent superposition and entanglement of quantum confined states, which have attracted much attention over the years [89-112]. Recently, researchers have successfully realized the relativistic arti-

ficial molecule by using two coupled Klein GQDs with relaticistic Dirac fermions. The bonding and antibonding states are detected and imaged in the relativistic artificial molecule by STM measurements [113].

Figure 7(a) schematically shows the relativistic artificial molecule with the fomation of bonding and antibonding states. The two adjacent Klein GQDs, which have the almost identical size [Fig. 7(b)], grown via CVD method are selected to form the relativistic artificial molecule states. Taking high-resolution $\mathrm{d} I / \mathrm{d} V$ spectra at different positions of the two coupled Klein GQDs [Figs. 7(d), (e)], the lowest quasi-bound state is observed to split into two peaks with the seperation of about $30 \mathrm{meV}$ [113]. Such a splitting is attributed to the fomation of molecular states, i.e., the bonding states $\sigma$ with lower energy and antibouding states $\sigma^{*}$ with higher energy [89-112]. It is well known that the lowest quasi-bound state is not well-confined since it distributed in the center of dot without forming a WGM, while the quasi-bound states with higher energy and higher angular momentum are much better confined in the Klein GQDs because of the formation of WGM resonances $[8,15,35]$. Therefore, the coupling strength reflected by the splitting energy is strongest in the lowest quasi-bound state and decreases with increasing the energy of quasi-bound states, as shown in Fig. 7(c). The LDOS map across the two coupled dots shown in Fig. 7(f) also directly reveals the formation of bonding states $\sigma$ and antibounding states $\sigma^{*}$ due to the coupling [113]. The relativistic molecular states are further explored in magnetic fields. It is intersting to find that the bonding and antibonding states gradually split into four peaks with increasing magnetic field, as marked by $\sigma_{+1 / 2}^{*}, \sigma_{-1 / 2}^{*}, \sigma_{+1 / 2}, \sigma_{-1 / 2}$ in Fig. $7(\mathrm{~g})$ [113]. Here, $\pm 1 / 2$ marks the angular momentum number. And the splitting energy between $\sigma_{+1 / 2}^{*}\left(\sigma_{+1 / 2}\right)$ and $\sigma_{-1 / 2}^{*}\left(\sigma_{-1 / 2}\right)$ increases linearly with increasing magnetic field. This kind of large splitting of bonding states and antibonding states in magnetic fields are attributed to the lifting of angular momentum $\pm m$ of the quasi-bound states, because that external magnetic field bends the trajectory of charge carriers and breaks the time inversion symmetry of the Klein GQDs [12, 31, 40, 42, 43, 59].

In summary, the relaticisitc artificial molecule is realized by coupling two Klein GQDs with the formation of bonding and antibonding states. Such a relaticisitc artificial molecule may have further application in quantum information processing.

\section{Bound states in GQDs}

Althrough many breakthroughs have been achieved in QDs formed in semiconductor heterostructures based on GaAs [2-5], the spin decoherence in these QDs shortens the coherence time and limits their appplication to realize 
(a)

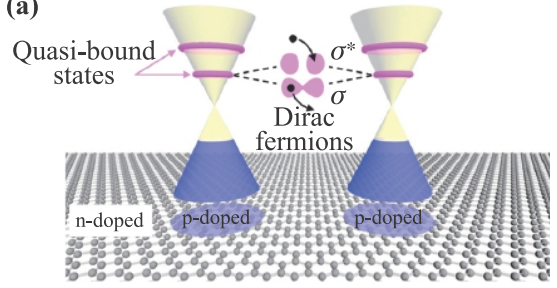

(c)

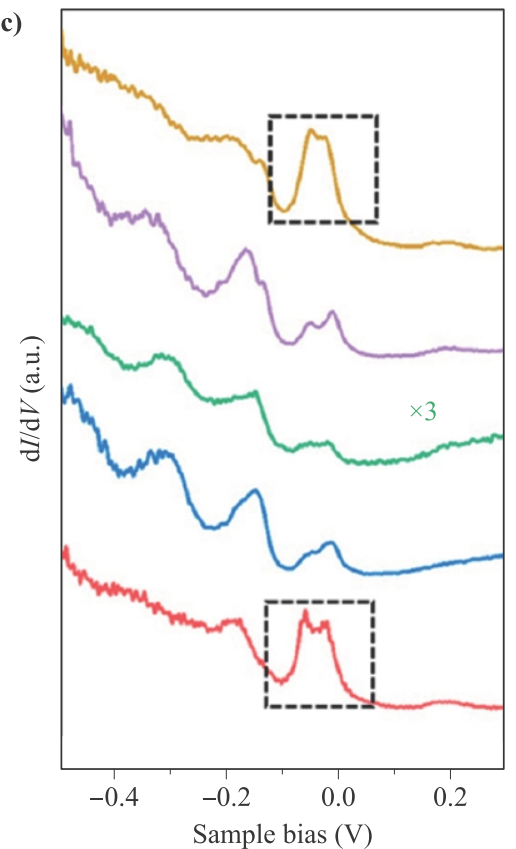

(b)

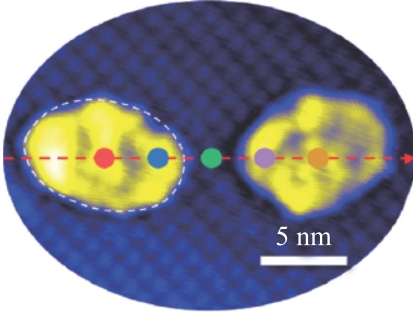

(d)
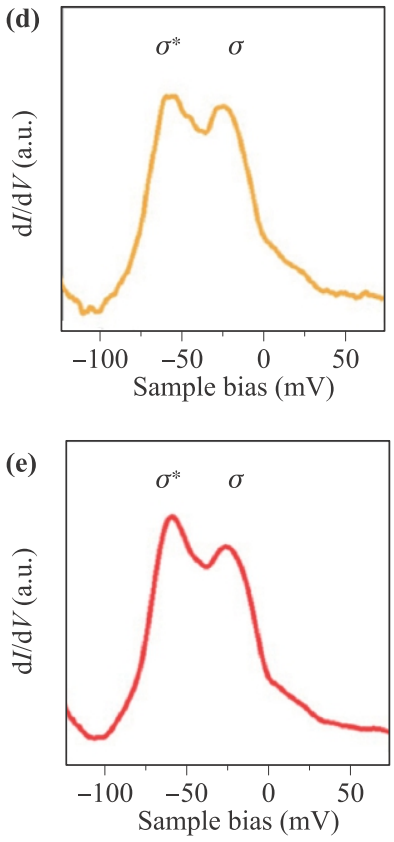

(f)

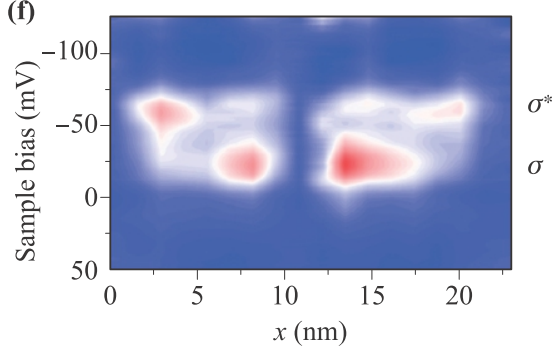

(g)

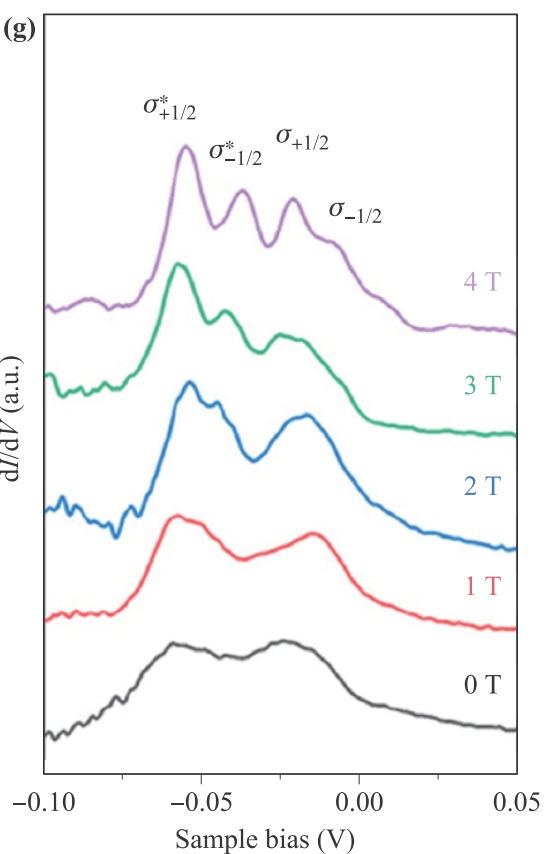

Fig. 7 (a) Schematic of relativistic artificial molecule realized by two coupled Klein GQDs. (b) STM image of two coupled Klein GQDs. (c) $\mathrm{d} I / \mathrm{d} V$ spectra with quasi-bound states taken at the different positions of the dot in panel (b). (d, e) Highresolution $\mathrm{d} I / \mathrm{d} V$ spectra acquired at the center of the two dots in panel (b), only showing the first quasi-bound state. (f) LDOS map of the first quasi-bound states taken along the dashed line in panel (b). (g) High-resolution $\mathrm{d} I / \mathrm{d} V$ spectra of the first quasi-bound state taken at the center of the left dot in different magnetic fields [113].

good qubits. Graphene owns weak spin-orbital coupling and weak hyperfine interaction which effectively weaken the spin decoherence effect, therefore, becomes an excellent candidate for spin qubit [6]. In recent years, many efforts have also been made on realizing "real" GQDs with bound states because of the potential application on spin qubit [13, 16-18, 22-24, 114-119].

The appearance of single-electron charging phenomenon is treated as the clear signature of that the studied dot is isolated from the surrounding area by tunnel barrier $[18,114,120-125]$. In order to trigger the singleelectron charging phenomenon and realize an isolated dot, the resistance of the tunnel barrier should be much larger than the quantum resistance $\left(R_{q}=h / e^{2}\right)$, which ensures that the wave function of electrons inside the dot is well localized [114]. Researchers have used several methods to realize bound states by forming GQDs. In transport measurents, researchers carved graphene layer into a desired geometry by using electron-beam lithography, then real- ized GQD devices with different sizes [18]. Their transport measurements found that the GQDs at large sizes (> 100 nanometers) exhibit periodic Coulomb blockade (single-electron charging) peaks and behave as conventional single-electron transistors. However, for the GQDs smaller than 100 nanometers, the single-electron charging peaks become strongly nonperiodic and the statisitic of these random peak spacing is well described by the theory of chaotic neutrino billiards, which indicate a major contribution of quantum confinement [18]. Based on these results, it was believed that the single-electron charging pheonomenon could not be observed in continuous graphene sheet. However, scientists still try to explore different methods to achieve trapping Dirac fermions in continuous monolayer graphene.

First, the nanoscale GQDs with bound states in a continuous graphene layer is realized by the strong coupling between graphene and the substrate at the circle boundary of the dot [114]. Second, combining electrostatic poten- 
(a)

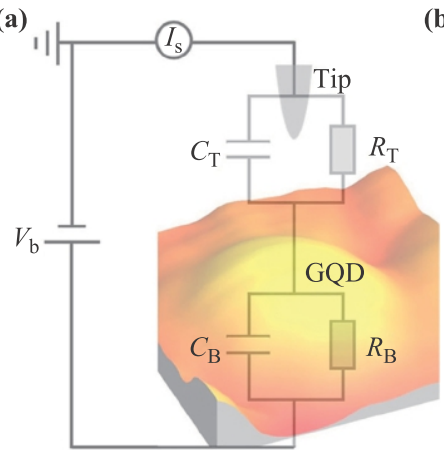

(b)

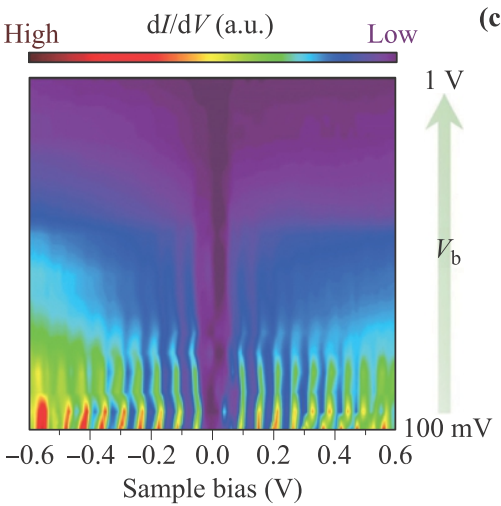

(c)

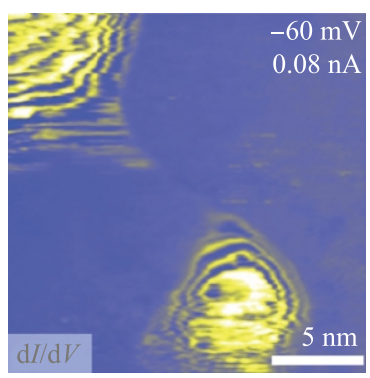

Fig. 8 (a) A typical GQD in a continuous graphene sheet, which forms a double-barriers tunnel junction with STM tip. The background image shows the single-electron charging system. (b) STS spectra map of a GQD taking by varying the bias voltage, i.e., the tip-sample distance. (c) $\mathrm{d} I / \mathrm{d} V$ map with fixed bias voltage $-60 \mathrm{mV}$ taken around the GQDs [114].

tials and energy gaps could also build GQDs with bound states in continuous graphene layer, which always lead to single-electron charging effects $[16,17,22-24,126]$. Surprisingly, the quadruplets of charging peaks resulting from bound states in tip-induced GQDs could be applied to study the degeneracy and broken symmetry states in different graphene systems [13].

\subsection{GQDs in continuous graphene sheet via strong coupling of substrate}

The strong graphene-substrate coupling could play a vital role in forming GQDs in continuous graphene sheet. Qiao et al. [114] grew the monolayer graphene on molybdenum foil by the CVD method. A reconstructed $\mathrm{Mo}_{2} \mathrm{C}$ surface forms underneath the continuous monolayer graphene, consisting of quantum dot-like vacancy islands and nanoscale islands. When the monolayer graphene was placed on the nanoscale vacancy islands, the $\pi$ orbital of the graphene is hybridization with the $d$ orbital of the Mo atoms adjacent to the boundary of the vacancy islands, and these $\pi$ electrons near boundary become strongly localized. Therefore, the electrons of the suspended graphene region over the vacancy island are obviously strongly confined and this region behaves as an isolated GQD in continuous graphene sheet [114].

Figure 8(a) shows the typical GQD in continuous graphene sheet realized by the strong graphene-Mo coupling. The spectra recorded outside the dot are Vshaped. However, the single-electron charging peaks are detected in the GQD region, which reflects that the GQD is electronically isolated from the surrounding continuous graphene sheet by the circular boundary [14, 18, 120125]. When the STM tip is placed above the GQD, a double-barrier tunnel junction is formed [Fig. 8(a)]. Considering the ohmic resistor between the GQD and the surrounding region $R_{B}$ remains a constant in the experiment, the ohmic resistor between the STM tip and the GQD
$R_{T}$ could be adjusted by varying the tip-GQD distance. An evolution of the spectra from the Coulomb blockade regime to the Coulomb staircase regime is obviously observed in STS spectra map of Fig. 8(b) by varying the bias voltage. Such a phenomenon could be described well by the simulation based on Coulomb blockade theory $[114,127,128]$. Figure $8(\mathrm{c})$ shows the spatial STS map at fixed bias voltage around the GQDs, which presents striking concentric rings inside the GQDs. Here, each ring in the map corresponds to a single Coulomb oscillation of the GQD [88, 120, 125, 129, 130].

With the strong graphene-substrate coupling, bound states are realized in nanoscale QGDs in continuous monolayer graphene, which pave a new way to electronically isolate graphene nanostructures in a continuous graphene sheet [114].

\subsection{STM tip-induced edge-free GQDs}

The STM tip induced edge-free GQDs are formed by combining the electrostatic potential of the STM tip with the external magnetic fields $[16,17,22,23,126]$, as schematically shown in Figs. 9(a) and (b). The external magnetic fields quantize the continuous band stucture of graphene into discrete LLs [58, 71, 131-134]. The probing STM tip, acting as a moveable top gate, bends the LLs of the region beneath the tip into the gaps between the LLs of the surrounding regions, which leads to an edge-free GQD beneath the tip with confined orbital states $[16,17,22,23,126]$. The gaps between the LLs help to achieve the bound-state confinement without resorting to physical edges, therefore, the four-fold (valley and spin) degeneracy of pristine graphene is preserved in the confined orbital states in the edge-free GQD. And each orbital state of the GQD could be occupied by four electrons. Because of the small capacitance $C$ of the edge-free GQD, evergy single excess electron on the GQD needs to overcome the electrostatic energy $E_{c}=e^{2} / C$ of the oc- 
(a)

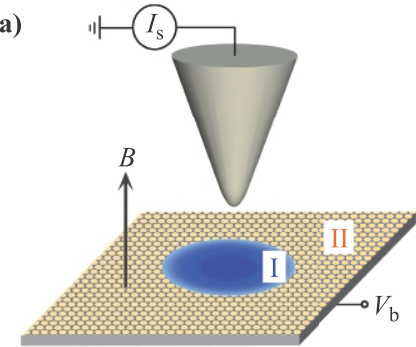

(b)

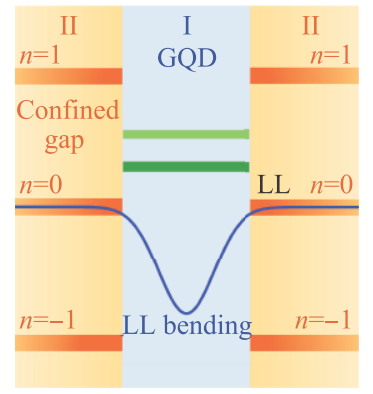

(c)

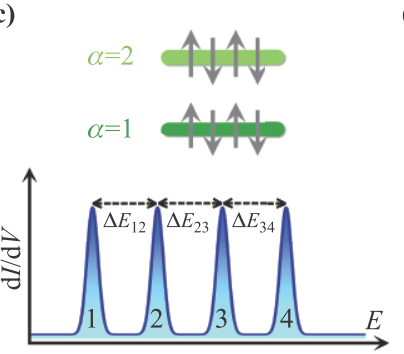

(d) (1) (3)

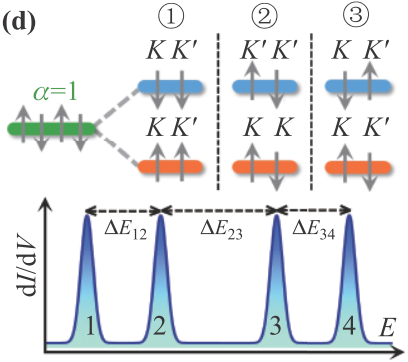

(e)

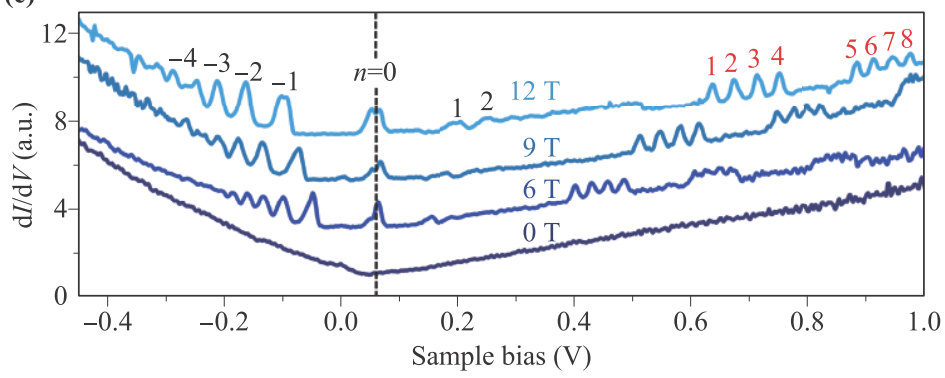

Fig. 9 (a) Schematic of the STM measurement on pristine grapene monolayer in external magnetic fields. (b) Schematic of STM tip-induced edge-free GQDs on pristine monolayer graphene. (c) Upper panel: Schematic confined orbital states in the tip-induced GQD on pristione graphene marked by $\alpha$. The arrows in opposite direction make the spin degrees of freedom in graphene. Lower panel: Schematic for the equal-spaced quadruplet of charging peaks in the STS spectrum of pristine graphene. $\Delta E_{12}, \Delta E_{23}$ and $\Delta E_{34}$ are the energy spacings of the four charging peaks. (d) Upper panel: Schematic confined orbital states in the tip-induced GQD on symmetry-broken graphene region. Lower panel: Schematic for the doublet charging peaks in the STS spectrum of symmetry-broken graphene region. $K$ and $K^{\prime}$ mark the two different valleys of graphene. (e) The experimental STS spectra of the pristine monolayer graphene in different magnetic fields with equal-spaced quadruplets of charging peaks in high bias voltage $[17,23]$.

cupied electrons during the STM measurement [Fig. 9(c)]. Consequently, a series of quadruplets of charging peaks are expected to be observed in the STS spectrum of pristine graphene $[16,17,22,23,126]$. Figure $9(\mathrm{e})$ shows the experimental STS spectra of pristine monolayer graphene with the equal-spaced quadruplets of charging peaks [17]. Each quadruplet of charging peaks corresponds to a confined orbital state and directly reflects the four-fold degeneracy of pristine monolayer graphene. When the spin/valley degeneracy of graphene is lifted, each quadruplet of charging peaks will be divided into two doublets as schematically shown in Fig. 9(d). Since this kind of edge-free GQDs is moveable with the STM tip, we could measure the symmetry broken states at any chosen position of graphene via detecting the quadruplets of charging peaks [17].

Different research groups have successfully used the STM tip-induced GQDs to detect the symmetry broken states in monolayer graphene[16, 17, 22, 23, 126]. $\mathrm{Li}$ et al. [17] used this method to detect the valley polarization and valley inversion in strained monolayer graphene [Fig. 10(a)]. Combining the strain-induced pseudo-magnetic field and the external magnetic field results in valley polarization and valley inversion of $N \neq 0$ LLs in strained graphene [135-143]. When the tip-induced edge-free GQD in strained monolayer graphene is formed during the STM measurement, the valley-polarized con- fined states are generated through confining the valleypolarized $N \neq 0$ LLs beneath the STM tip, and the doublets charging peaks are detected in the STS spectra at high bias voltage. The value of valley splitting $\Delta E_{V}$ could be obtained by measuring the energy spacings of the quartet charging peaks $\Delta E$ by using $\Delta E_{V}=$ $\Delta E_{23}-\Delta E_{12}\left(\Delta E_{34}\right)+2 E_{\mathrm{Z}}$, where $\Delta E_{12}, \Delta E_{23}$ and $\Delta E_{34}$ are the energy spacings of the first four charging peaks, $E_{\mathrm{Z}}$ is the Zeeman splitting. Here, the energy spacings of charging peaks $\Delta E$ are deduced from the voltage differences $\Delta V_{t i p}$ of the charging peaks in STS spectra by using $\Delta E=\eta e \Delta V_{t i p}$ with $\eta<1$ as the tip lever arm [143]. Figure 10(b) shows the energy map of $\left|\Delta E_{V}\right|$ as a function of positions in the strained graphene region. The white dashed lines mark the transition region where pseudomagnetic field is zero and there is no valley splitting. The directions of the pseudo-magnetic field and the valley polarization are both inverted when crossing this transition region. In this work, edge-free GQD method plays an essential role to study the valley-related phenomenon in strained graphene [143].

This method is also applied to investigate the broken symmetry states in defected graphene [23] and in moiré superlattices of graphene/hBN heterostructure [22], as shown in Figs. 10(c)-(f). The single-atom defects break the sublattice symmetry, and are expected to trigger novel 


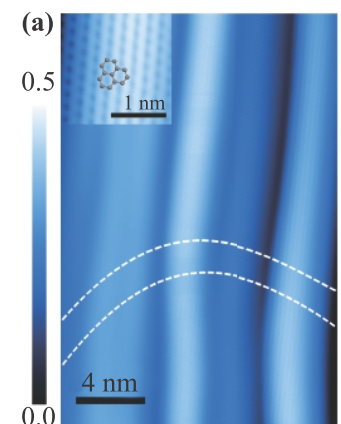

(b) 0.0

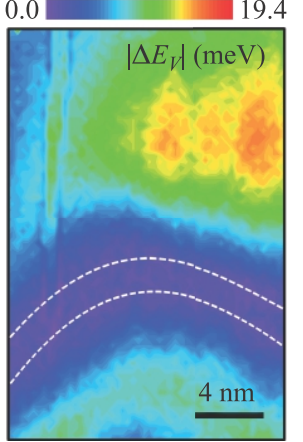

(c)

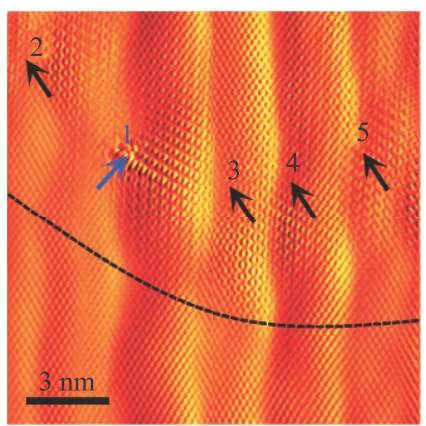

(d)

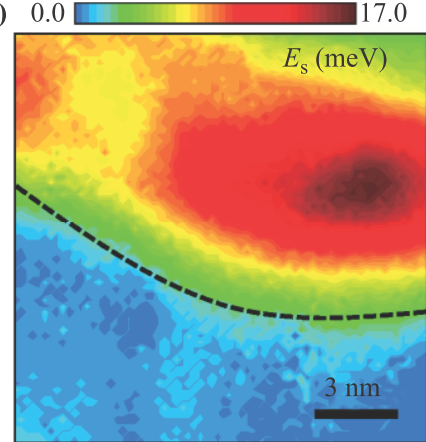

(e)

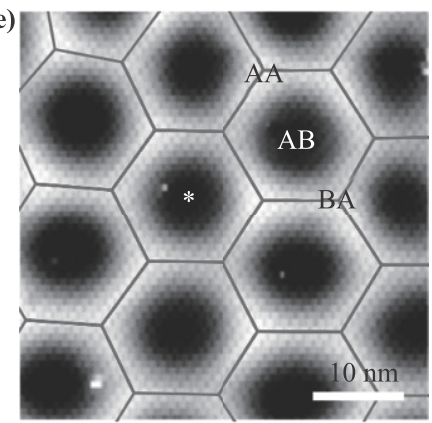

(f)

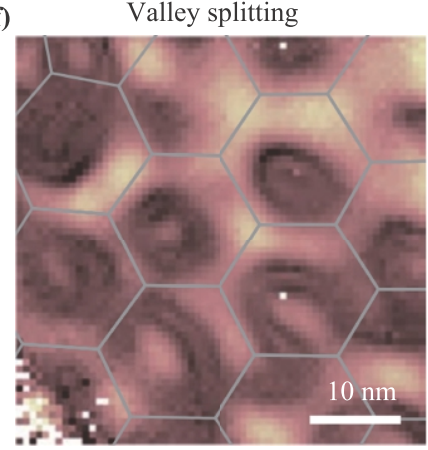

Fig. 10 (a) The STM image of a strained monolayer graphene grown on Rh foil [17]. The white dashed lines mark the transition region with pseudomagnetic field about $0 \mathrm{~T}$. Inset: The STM image with atomic-resolution in this area. (b) The absolute value of valley splitting $\left|\Delta E_{V}\right|$ as a function of positions in the strained graphene region of panel (a) [17]. In the transition region maked by white dashed lines, $\left|\Delta E_{V}\right|$ is about zero. (c) The STM image of a monolayer graphene area with several single-atom defects [23]. The arrows and numbers make the positions of the defects. (d) The energy map for the valley-dependent spin splitting $E_{\mathrm{s}}$ in the defect monolayer graphene region [23]. (e) STM image of moiré superlattices in graphene/hBN heterostructure [22]. (f) The energy map for the valley splitting of the same region in panel (e) [22].

broken symmetry states in graphene. Researchers have detected the valley-dependent spin splitting around the single-atom defects of graphene using the edge-free GQD method [23] [Figs. 10(c), (d)]. The energy map of $\Delta E_{\mathrm{s}}$ is acquired via measuring the quartet charging peaks in each position of the defected graphene region, as shown in Fig. 10(d), which reveals that the value of $\Delta E_{\mathrm{s}}$ has an obvious spatial extension around the defects. Freitag et al. have also used the edge-free GQD method to explore the broken symmetry states in moiré superlattices of graphene/hBN heterostructure [22], as shown in Figs. 10(e) and (f). From the spatial energy map of valley splitting in Fig. 10(f), the distribution of valley splitting follows the period of the moiré superlattices in graphene/hBN heterostructure. And they also find the valley inversion phenomenon inside each moiré superlattice. The valley polarization and valley inversion in graphene/hBN heterostructure are resulted from the strong interaction between the $\mathrm{hBN}$ substrate and the monolayer graphene. This work indicates that the graphene $/ \mathrm{hBN}$ heterostructure with moiré superlattices is an ideal platform to study valley manipulation physics.

Uing the STM tip-induced edge-free GQD to detect the broken symmetry states at nanoscale and single-electron accuracy is suitable not only for studying monolayer graphene, but also for studing other two-dimentional material systems. The above results provide a new and intuitive method to explore the broken symmetry states, and such a method is expexted to play a vital role when investigating the basic physical properties of two-dimentional materials in the future.

\subsection{Novel bound states in bilayer GQDs}

Graphene monolayer $\mathrm{p}-\mathrm{n}$ junction is an ideal paltform to investigate the novel confinement behavior of massless Dirac fermions, such as whispering gallery modes described in Section 2.1. Quite differenent from monolayer graphene, Bernal bilayer graphene can be gapped by applying a perpendicular electric filed which breaks its inversion symmetry [144-151]. This feature makes it possible to confine electrons in bilayer graphene by using electrostatic potentials with the formation of QD confinement states [152-154]. Transport measurements have detected the quantum confiment of massive Dirac fermions with Coulomb blockade oscillations in gate-defined bilayer GQDs [152, 153]. Jr Velasco et al. [154] have utilized STM-based technique to create the bilayer GQDs on hBN subatrate (see the second method of creating GQDs described in Section 2.1), and spatially maps the concentric rings with gate-tunable diameters that are explained 
(a)

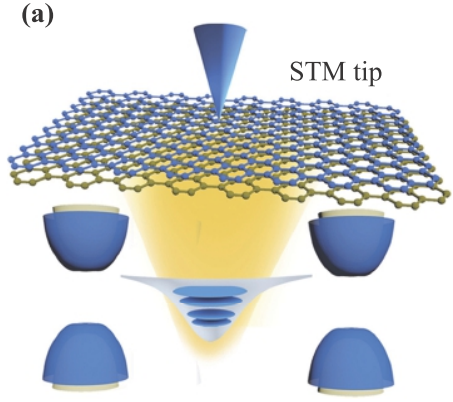

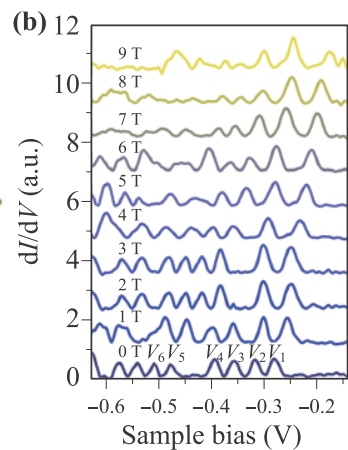

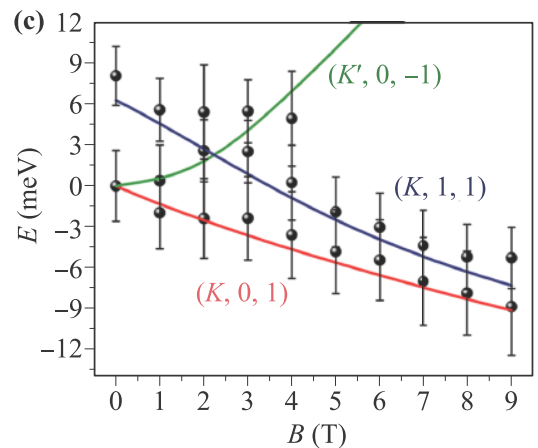

Fig. 11 (a) Schematic of the confined bound states in a edge-free bilayer GQD induced by conbining the electric field of STM tip and the bandgap of the Bernal bilayer graphene. (b) The series of single-electron charging peaks of the bound states in the edge-free bilayer GQD from $0 \mathrm{~T}$ to $9 \mathrm{~T}$. $V_{1}-V_{6}$ mark the voltage positions of the charging peaks. (c) Comparison of the experimental bound valley levels $\varepsilon_{1}$ and $\varepsilon_{2}$ with the theoretical valley levels $(K, 0,1),\left(K^{\prime}, 0,-1\right)$ and $(K, 1,1)[13]$.

by single-electron charging of localized states arising from the quantum confinement of massive Dirac fermions in bilayer GQDs. Additionally, bilayer GQDs have nontrivial band topology [155], controlable quantum degree of freedom $[156,157]$ and long decoherence time [6]. These special features are all favorable in quantum inforamtion researches, and theoretical works have predicted novel confinement behaviors of massive Dirac fermions in bilayer GQDs $[26,158]$. Researchers have detected the valley and Zeeman splittings with a spin $g$-factor $g_{s} \approx 2$ in single electron charging of gate-defined bilayer GQDs [156], and the valley splitting linearly depends on the external perpendicular magnetic field [156, 157]. Ge et al. [159] have directly visualized the wave function shape of bilayer GQD states with a robust broken rotational symmetry, which is contributed to the low energy anisotropic bands. And they also demonstrated that the nontrivial band topology of bilayer graphene could be manifested and manipulated by imaging quantum interference patterns in bilayer GQDs.

Specially, quite different from monolayer graphene where the Berry phase can only take two values $(0$ or $\pi)$, the Berry phase in gapped Bernal bilayer graphene can be continuously tuned from 0 to $2 \pi[51,53-55,144,160$ 166], which offers a unique opportunitity to study the effect of continuous tunable Berry phase on physical phenomenon [13, 163]. In 2020, Liu et al. [13] reported the Berry-phase-switched valley splitting and crossing in bilayer GQDs. In zero magnetic field, the moveable edgefree bilayer GQD is realized by conbining the electric field of STM tip and the gap of Bernal bilayer graphene as schematically shown in Fig. 11(a) [13, 16, 17, 22, 23]. A series of single electron charging peaks resulted from the cofined bound states in the edge-free bilayer GQD are detected in the STS spectra, and the first four peaks correspond to the lowest bound state [Fig. 11(b)]. With external perpendicular magnetic fields, the Berry phase of the bound states in the bilayer GQD is changed from zero to $2 \pi$ continuously, and also results in the Berry phase difference in the two inequivalent valleys in the bilayer graphene [163]. Therefore, the gaint valley splitting and crossing of the lowest bound state in the bilayer GQDs are observed by detecting the doublet charging peaks in the STS spectra at different magnetic fields, which are accorded with theoretical calculation [Figs. 11(b), (c)]. The result of valley manipulation at single-electron level in this work suggests that the gapped Bernal bilayer graphene is an excellent platform to explore Berry-phase tunable phenomenon, and the sensitive magnetic-field-controled valley switch could be realized in bilayer GQDs [13].

The novel confinement states, including the manipulation of Berry curvature and valley pseudospin in bilayer GQDs, inspire future works of using confinement states to study the properties of two-dimentional materials that have nontrivial Berry curvature, such as semiconducting transition metal dichalcogenides (TMDs) [167], topological insulators [168], and Weyl semimetals [169-171]. In addition, the technical advancements in bilayer GQDs works are also used to fabricate coupled double and multiple bilayer GQDs applied to realize the quantum bits based on valley and spin degrees of freedom $[172,173]$.

\section{Edge-terminated confinement in triangular zigzag nanographenes}

Creating edge-terminated patterned nanographenes is another direct way to realize novel quantum confinement in graphene, which may also introduce interesting magnetic structures. The electronic and magnetic properties of the nanographene structures strongly depend on their shape, size and topology [19]. Among the nanographenes, triangular zigzag nanographenes generated by the fusion of benzenoid rings in triangular fashion (known as nonKekulé polynuclear benzonoid compounds) are quite special and interesting, because they are predicted to hold multiple unpaired $\pi$-electrons and an increasing groundstate total spin quantum number $S$ with the increase of size according to Ovchinnikov's rule [174-180]. Owning 
to their intrinsic high-spin magnetic ground states for molecular spintronics, triangulene zigzag nanographenes, such as triangulene, $\pi$-extended triangulene and triangulene dimers [Fig. 1(c)], have attracted widespread attention both in fundermental researches [181-183] and in future information technologies [176, 184, 185]. Recent years, researchers have successfully synthesized unsubstituted triangulene [186], $\pi$-extended triangulene $[19,21]$ and triangulene dimers [20] using different methods or different precursors. And their structural characteriztion, as well as their corresponding frontier molecular orbital states and Coulomb gaps are directly detected by highresolution STM measurements and noncontact atomic force microscopy (nc-AFM) measurements [19-21, 186].

\subsection{The properties of triangulene and $\pi$-extended triangulene}

Triangulene is the smallest triplet-ground-state polybenzenoid (six fused rings, $S=1$ ). The addition of substituents has made it possibele to synthesize and stabilize the trangulene core with verifying the triplet ground state by electron paramagnetic resonance spectroscopy [187189], but the synthesis and characterization of unsubstituted trianglene was lacking. In 2017, a new tip-assisted atomic manipulation method via a combined scanning tunelling and atomic force microscope (STM/AFM) was used to synthesize unsubstituted triangulene and detect its structural and electronic properties [186]. The precursor compound was deposited on $\mathrm{Cu}(111), \mathrm{NaCl}(100)$ and $\mathrm{Xe}(111)$ surfaces, then they dehydrogenated the precursors to obtain triangulene by positioning the tip above the precursor and manipulating the bias voltage. The obtained triangulenes are directly imaged by the AFM function and STM function as shown in Figs. 12(a) and (b), respectively [186]. The AFM image in Fig. 12(a) shows the detailed strutral characterization of triangulene and confirms the $C_{3}$ symmetry of the molecular structure. The inner and outer carbons appear less-pronounced differences that corroborate a planar molecular structure [Fig. 12(a)]. The STM image in Fig. 12(b), which is accorded with the simulated STM image calculated by assuming an extended $s$-like wavefunction of the tip [190], confirms that the triangulenes are unsubstituted with two unpaired electrons [186].

Assuming a ferromagnetic alignment of the two unpaired electrons (triplet ground state) in spin-polarized DFT calculation, the resulted quasipartical energy levels of the triplet states are schematically shown in Fig. 12(c) [186]. Two pairs of non-disjoint degenerate orbitals $\psi_{2}, \psi_{3}$, i.e., an occupied pair (spin up) and an unoccupied pair (spin down) in Fig. 12(b), correspond to the frontier molecular orbitals. Figure 12(d) shows the $\mathrm{d} I / \mathrm{d} V-V$ spectra taken at the center of a triangulene on $\mathrm{Xe}(111)$. The two pronounced peaks at $-1.4 \mathrm{~V}$ (a)

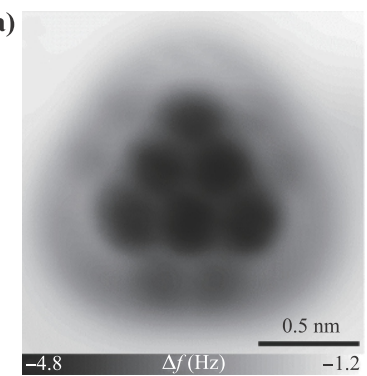

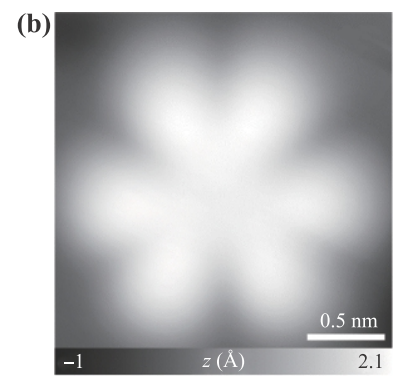

(c)
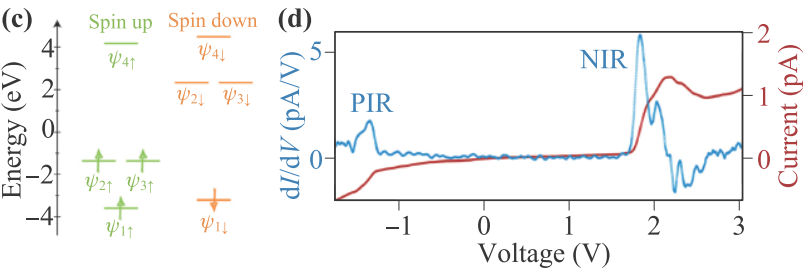

Fig. 12 (a) AFM image of a triangulene on Xe. (b) STM image at the voltage corresponding to the negative ion resonance (NIP). (c) Schematical energy-levels in spin-polarized DFT calculation. (d) STS measurement of a triangulene on Xe with $I-V$ curve (red) and $\mathrm{d} I / \mathrm{d} V-V$ curve (blue). The $\mathrm{d} I / \mathrm{d} V-$ $V$ spectrum shows the positive ion resonance (PIR) peak and the NIR peak, respectively [186].

and $1.85 \mathrm{~V}$ correspond to the positive and negative ion resonance, and reveal the broad gap of about $3.25 \mathrm{~V}$ arising from the Coulomb energies. This gap is quite larger than the pure Coulomb gap for a system with comparable size [191], which indicates there is a significant spin splitting of triangulene [186]. The tip-assisted method in this work is also proved to be an effective way to manipulate the structure or property of $2 \mathrm{D}$ materials, which is widely used in other researches until now [134, 192-194].

The successful study of triangulene has inspired researchers to synthesis and invesitgate its larger homologues, that is, $\pi$-extended triangulene, such as $\pi$-extended [4]- and [5]-triangulene consisting of ten and fifteen fused rings with $S=3 / 2$ and 2 , respectively. Recently, researches have obtained $\pi$-extended [4]- and [5]-triangulene on metal and insulator surfaces through a combined insolution and on-surface synthesis method $[19,21]$. Comparing with the above tip-assisted approach which only manipulates one target molacular at one time, the bottomup on surface synthetic approach has a great potential to fabricate atomically precise graphene-based nanostructure with large scalability [195-202]. In order to synthesize larger homologues of zigzag-edged triangulenes with large net spin, it is always necessary to design suitable molecular precursors and synthetic routes. The structural characterization of the obtained $\pi$-extended [4]- and [5]-triangulene is directly visualized at submolecular resolution by STM or nc-AFM with a CO-functionalized tip [203-206], as shown in Figs. 13(a) and (b). The triangle-shaped molecular consisting of fused benzene rings with zigzag edges could be clearly observed $[19,21]$. STS measurements are 

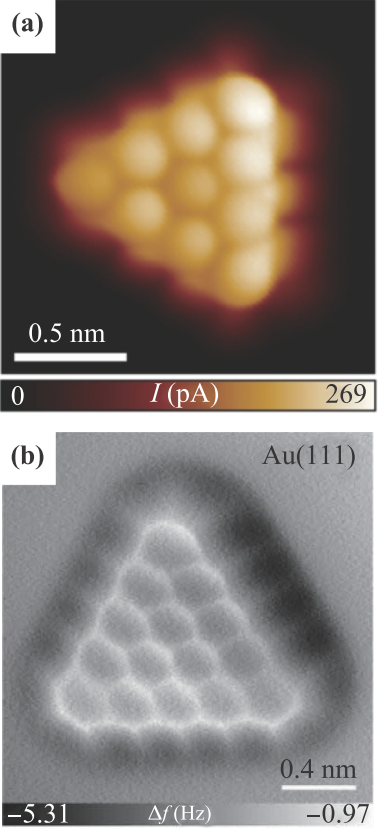
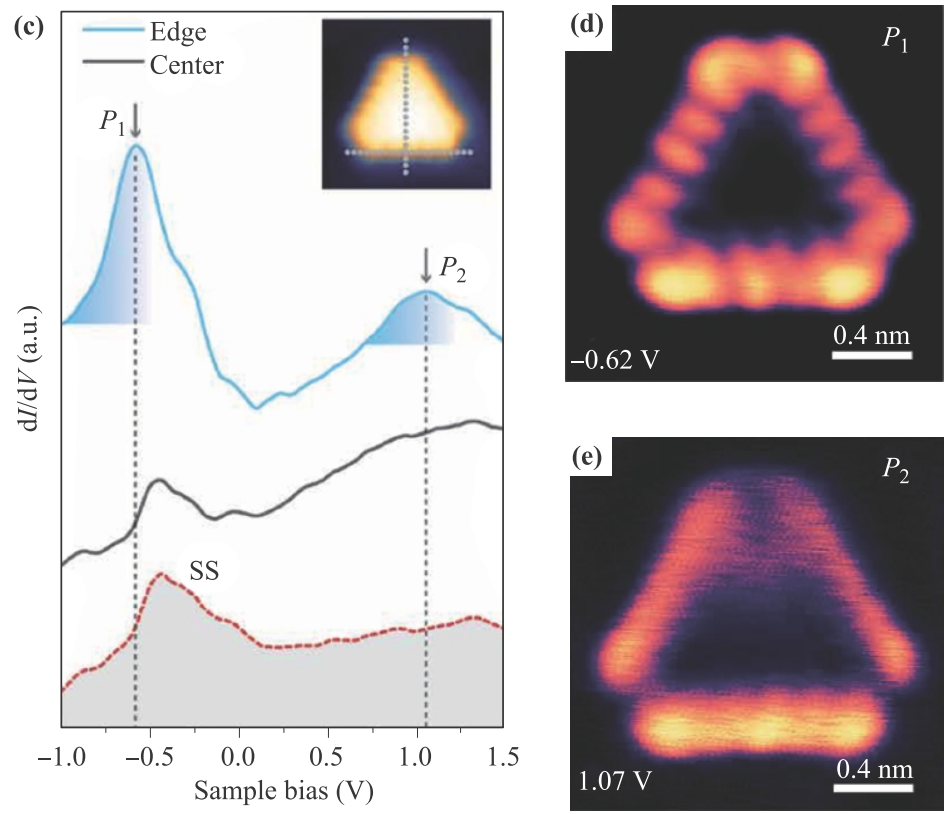

Fig. 13 (a) Ultrahigh-resolution STM image of a $\pi$-extended [4]-triangulene on weakly interacting Au(111) with a COfunctionalized tip [19]. (b) nc-AFM image of a $\pi$-extended [5]-triangulene on $\mathrm{Au}(111)$ using a CO-functionalized tip [21]. (c) $\mathrm{d} I / \mathrm{d} V-V$ spectra taken at different sites of the [5]-triangulene and the Au(111) substrate [21]. (d, e) STS maps taken at energy positons of $-0.62 \mathrm{~V}$ and $1.07 \mathrm{~V}$, respectively [21].

carried out to probe the frontier molecular orbitals of $\pi$ extended triangulene, and then compared with theoretical calculations. Here, we take the result of $\pi$-extended [5]triangulene as an example. Figure $13(\mathrm{c})$ shows $\mathrm{d} I / \mathrm{d} V-V$ spectra acquired at the center and edge of a triangulene and at the bare $\mathrm{Au}(111)$ substrate surface. [21]. The two prominent peaks at around $-0.62 \mathrm{~V}\left(P_{1}\right)$ and $1.07 \mathrm{~V}\left(P_{2}\right)$ are only observed at the zigzag edges of [5]-triangulene, reflecting that the two peaks are associated with molecular states [21]. In STS maps at the energies of $P_{1}$ and $P_{2}$ states [Figs. 13(d), (e)], it is much obvious that $P_{1}$ and $P_{2}$ states only localized at the edge represented by a nodal patterns. A small difference is that the spatial distribution of $P_{2}$ state shows a slightly blurred nodal structure. These features are accorded with the theoretical results calculated by spin-polarized DFT method. The experimenal $P_{1}$ and $P_{2}$ states correspond to the spin-polarized edge states [21].

The successful synthesis of the high-spin, delocalized triangulene and $\pi$-extended triangulene not only enrichs the magnetic transport properties at single-molecular level, but also paves a new way to build larger triangular zigzagedged GQDs with atomic precision for quantum electronic and spintronic devices.

\subsection{The properties of triangulene dimers}

Based on their high-spin ground states, many interesting fundamental and technological prospects may be triggered by the construction of one-dimensional chains and two-dimensional networks incorporating triangular zigzag nanographenes as building blocks, such as elusive quantum states [207] and room-temperature long-range magnetic order [208-210]. The bottom-up on surface synthetic approach, as a chemical tool box, makes it possible to fabricate extended triangular zigzag nanographenes with designed proper molecular precursors and synthetic routes [195-200]. In 2020, Mishra et al. [20] successfully synthesize covalently bonded triangulene dimers, including two triangulene units connected through their minority sublattice atoms [triangulene dimer $1, S=0$, Fig. 14(a)] and two triangulene dimer with 1,4-phenylene spacer [triangulene dimer 2, $S=0$, Fig. 14(b)] via a combined in-solution and on-surface synthesis method, and studied their magnetic properties via STS and STM-based inelastic electron tunneling spetroscopy (IETS).

Figures 14(c) and (d) are the ultrahigh-resolution STM images of triangulene dimer 1 and triangulene dimer 2 with a CO-functionalized tip, respectively, clearly showing their bond-resolved structure. Considering the e- $\mathrm{e}$ interaction within the mean-filed Hubbard model (MFH), the zero-energy states of triangulene dimers 1, 2 are lifted to form the singly occupied molecular orbitals (SOMOs) and singly unoccupied molecular orbitals (SUMOs) with opening a sizeable Coulomb gap [Fig. 14(e)] [20]. And $\mathrm{MFH}$ calcaulation also predicts an antiferromagnetic order between the triangulene units of dimer 1 and dimer 2 which leads to an $S=0$ open-shell singlet ground state. The spin-split frontier molecular orbital states and their corresponding Coulomb gaps about $1.65 \mathrm{~V}$ of the triangu- 
(a)

$$
\begin{aligned}
& \text { Triangulene dimer } \\
& N_{\mathrm{A}}=N_{\mathrm{B}}=22, S=0
\end{aligned}
$$

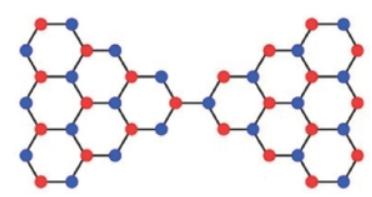

(c)

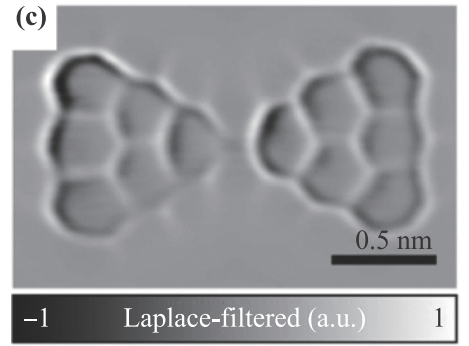

(b)
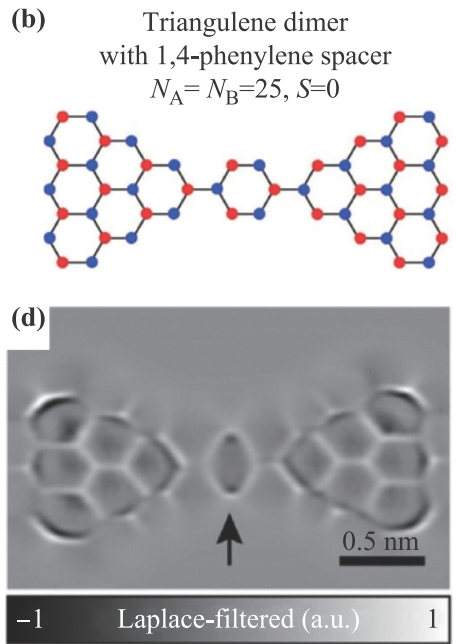

(e)

Spin up/spin down

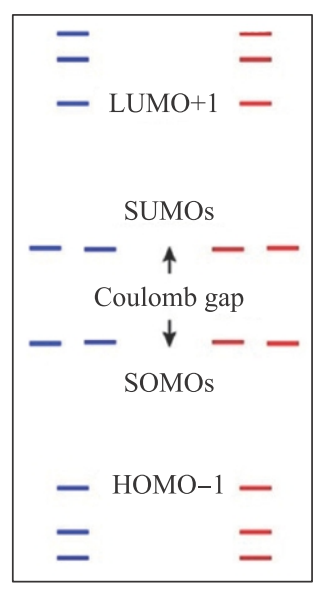

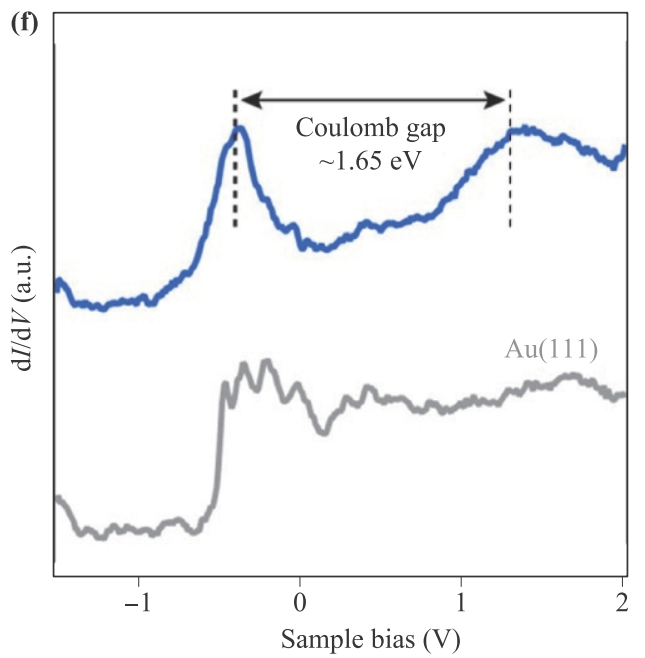

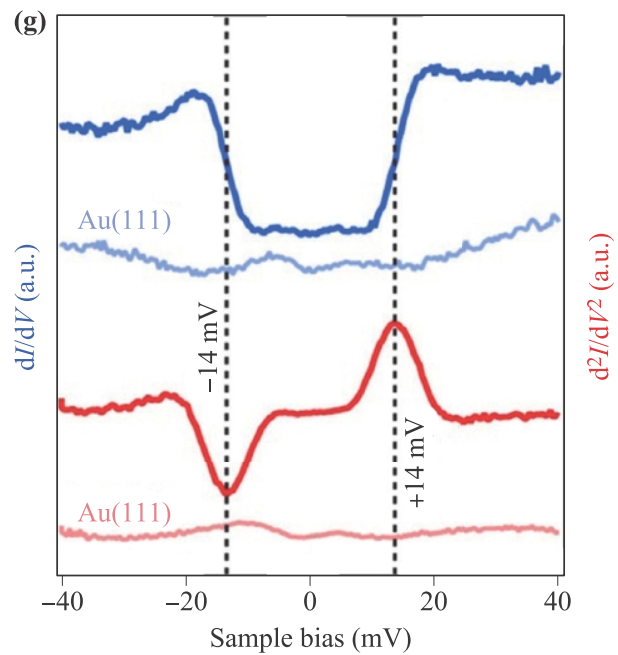

Fig. 14 (a, b) Schematical structures of two triangulene units connected through their minority sublattice atoms (triangulene dimer 1) and two triangulene dimer with 1,4-phenylene spacer (triangulene dimer 2), respectively. (c, d) The corresponding laplace-filtered ultrahigh-resolution STM images of triangulene dimer 1 and triangulene dimer 2 with a CO-functionalized tip, respectively. (e) Theoretical energy spectrum of triangulene dimer 1 along with the spin polarization plot calculated by meanfiled Hubbard model. (f) Long-range $\mathrm{d} I \mathrm{~d} V-V$ spectra acquired on triangulene dimer 1 and on $\mathrm{Au}(111) \mathrm{surface}$. (g) $\mathrm{d} I \mathrm{~d} V-V$ spectra (blue solid curve) and IETS spectra (red curve) taken on triangulene dimer 1 and on Au(111) surface in the vicinity of the Fermi level [20].

lene dimers are detected by STS spectra [Fig. 14(f)] and STS maps [20]. These results are quite similar with that of $\pi$-extended triangulenes in Section 4.1.

$\mathrm{d} I / \mathrm{d} V-V$ spectrum on triangulene dimer 1 in the vincinity of the Fermi level shows the conductance steps symmetric around zero bias [Fig. 14(g)], indicating the existence of inelastic excitation $[134,211,212]$. The excitation threshold was extracted to be about $\pm 14 \mathrm{mV}$ in the IETS spectrum through fitting with the antiferromagnetic spin-1 Heisenberg dimer model [213]. Here, the inelastic excitation phenomenon is resulted from singletriplet $(S=0$ to $S=1)$ spin excitation, and the strength of effective exchange coupling $J_{\text {eff }}$ (or the singlet-triplet gap) of triangulene dimer 1 is directly measured to be $14 \mathrm{meV}$. The $J_{\text {eff }}$ of triangulene dimer 2 is detected to be about $2 \mathrm{meV}$ using the same method [20]. The difference of $J_{\text {eff }}$ between triangulene dimer 1 and dimer 2 demonstrates the tunability of intertriangulene magnetic coupling in triangular zigzag nanographenes. The organic spacers in the dimer structure could tune the magnetic coupling and correlations between the triangulene units. Therefore, different nanoarchitectures based on triangular zigzag nanographenes could further be designed and synthesized with tunable coupling strengths and magnetic ground states.

Based on the bottom-up on surface synthetic approach and STM/AFM measurements, many other different nanographenes with special structures are also formed and investigated besides the above triangular zigzag nanographenes, such as Clar's goblet [214] and 
[7]triangulene quantum rings [215]. The Clar's goblet shows a rubost antiferromagnetic order with the exchangecoupling strenght about $23 \mathrm{meV}$, which is comparable to the reported values in previous researches of graphene nanoribbon junctions [216] and triangulene dimer [20]. And the magnetic ground states of Clar's goblet could be switched on/off by using tip-assisted atomic manipulation method [214]. These rich nanographenes with special structures and high-spin magnetic ground states open up a fantastic way to design and fabricate next-generation carbon-based quantum devices.

\section{Conclusion and perspectives}

We have reviewed the recent progresses of GQDs studies mainly using STM and STS measurements. According to the confinement strength, the GQDs are divided into Klein GQDs, bound-state GQDs and edge-terminated GQDs in this review. The whispering galley mode in Klein GQDs fully reflects the relativistic property of quasiparticles in graphene, which consequently results in relativistic artifical molecular by two coupled Klein GQDs. In the researches of Klein GQDs, external perpendicular magnetic fields are used as an effective manipulation approach to trigger and control the novel properties by tuning Berry phase and e-e interaction, such as on/off berry phase switch and quantum Hall wedding cake-like structure. In bound-state GQDs, we have discussed several different methods to realize bound states, including using strong coupling effect of substrate and tip-assisted approach. The tip-induced edge-free GQDs also serve as an intuitive mean to explore the broken symmetry states at nanoscale and single-electron accuracy, and are expected to be used in studying physical properties of other twodimentional materials. Finally, novel magnetism properties are induced in GQDs by synthesizing edge-terminated nanographenes, such as triangulene, $\pi$-extended triangulene and triangulene dimers, where the high-spin magnetic ground states are resulted from the zigzag-terminated edges. Based on the successful construction and the deep investigation of different GQDs with special structures and unconventional properties, a rich tool box of GQDs is realized for future fundamental researches and nextgeneration carbon-based quantum devices.

In the meanwhile, there are still many attractive topics in the researches of GQDs. For example, (i) the confinement states in GQDs are closely dependent on the confining potential barrier, including the deepth, the width and the sharp of potential barriers. Different confining potentials have realized quasi-bound states of weak confinement and bound states of strong confinement in graphene. If the confining potential barrier could be accurately controled in the experiment, new confinement states and novel critical effects will be detected in GQDs. (ii) The magnetism of triangulene and its dimers originates from their specail zigzag edges. Synthesizing different edgecontrolable GQDs will trigger much more intersting confinement states related to magnetic properties. Theoretical and experimental efforts are also needed to investigate the magnetic ground states and excited states in the GQDs with designed edges. (iii) Besides of the study of single GQD, the synthesis and investigation of GQD arrays also attract much attention because of their potential application in quantum information technologies. The study of relativistic artificial molecules by two coupled Klein GQDs has provided us with a successful case in the research of GQD array. In the future, realizing different GQD arrays will introduce more interesting phenomena through manipulating the couplings between the QDs.

Acknowledgements This work was supported by the National Natural Science Foundation of China (Grant Nos. 11974050, 11674029, and 12104144), and the Natural Science Foundation of Hunan Province, China (Grant No. 2021JJ20025). L. H. also acknowledges support from the National Program for Support of Topnotch Young Professionals, support from "the Fundamental Research Funds for the Central Universities", and support from "Chang Jiang Scholars Program".

Open Access This article is licensed under a Creative Commons Attribution 4.0 International License, which permits use, sharing, adaptation, distribution and reproduction in any medium or format, as long as you give appropriate credit to the original author(s) and the source, provide a link to the Creative Commons license, and indicate if changes were made. The images or other third party material in this article are included in the article's Creative Commons license, unless indicated otherwise in a credit line to the material. If material is not included in the article's Creative Commons license and your intended use is not permitted by statutory regulation or exceeds the permitted use, you will need to obtain permission directly from the copyright holder. To view a copy of this license, visit http://creativecommons.org/licenses/by/4.0/.

\section{References}

1. D. Loss and D. P. DiVincenzo, Quantum computation with quantum dots, Phys. Rev. A 57(1), 120 (1998)

2. J. Elzerman, R. Hanson, L. W. Van Beveren, B. Witkamp, L. Vandersypen, and L. P. Kouwenhoven, Single-shot read-out of an individual electron spin in a quantum dot, Nature 430, 431 (2004)

3. R. Hanson, L. W. van Beveren, I. Vink, J. Elzerman, W. Naber, F. Koppens, L. Kouwenhoven, and L. Vandersypen, Single-shot readout of electron spin states in a quantum dot using spin-dependent tunnel rates, Phys. Rev. Lett. 94(19), 196802 (2005)

4. F. H. Koppens, C. Buizert, K. J. Tielrooij, I. T. Vink, K. C. Nowack, T. Meunier, L. Kouwenhoven, and L. Vandersypen, Driven coherent oscillations of a single electron spin in a quantum dot, Nature 442(7104), 766 (2006) 
5. J. R. Petta, A. C. Johnson, J. M. Taylor, E. A. Laird, A. Yacoby, M. D. Lukin, C. M. Marcus, M. P. Hanson, and A. C. Gossard, Coherent manipulation of coupled electron spins in semiconductor quantum dots, Science 309(5744), 2180 (2005)

6. B. Trauzettel, D. V. Bulaev, D. Loss, and G. Burkard, Spin qubits in graphene quantum dots, Nat. Phys. 3(3), 192 (2007)

7. J. Lee, D. Wong, J. F. Jr Velasco, S. Rodriguez-Nieva, H. Z. Kahn, T. Tsai, K. Taniguchi, A. Watanabe, Zettl, F. Wang, L. S. Levitov, and M. F. Crommie, Imaging electrostatically confined Dirac fermions in graphene quantum dots, Nat. Phys. 12(11), 1032 (2016)

8. C. Gutiérrez, L. Brown, C. J. Kim, J. Park, and A. N. Pasupathy, Klein tunnelling and electron trapping in nanometre-scale graphene quantum dots, Nat. Phys. 12(11), 1069 (2016)

9. P. G. Silvestrov and K. B. Efetov, Quantum dots in graphene, Phys. Rev. Lett. 98(1), 016802 (2007)

10. K. K. Bai, J. J. Zhou, Y. C. Wei, J. B. Qiao, Y. W. Liu, H. W. Liu, H. Jiang, and L. He, Generating atomically sharp $\mathrm{p}-\mathrm{n}$ junctions in graphene and testing quantum electron optics on the nanoscale, Phys. Rev. B 97(4), 045413 (2018)

11. Y. Jiang, J. Mao, D. Moldovan, M. R. Masir, G. Li, K. Watanabe, T. Taniguchi, F. M. Peeters, and E. Y. Andrei, Tuning a circular $\mathrm{p}-\mathrm{n}$ junction in graphene from quantum confinement to optical guiding, Nat. Nanotechnol. 12(11), 1045 (2017)

12. F. Ghahari, D. Walkup, C. Gutiérrez, J. F. RodriguezNieva, Y. Zhao, J. Wyrick, F. D. Natterer, W. G. Cullen, K. Watanabe, T. Taniguchi, L. S. Levitov, N. B. Zhitenev, and J. A. Stroscio, An on/off Berry phase switch in circular graphene resonators, Science 356(6340), 845 (2017)

13. Y. W. Liu, Z. Hou, S. Y. Li, Q. F. Sun, and L. He, Movable valley switch driven by Berry phase in bilayergraphene resonators, Phys. Rev. Lett. 124(16), 166801 (2020)

14. Z. Q. Fu, K. K. Bai, Y. N. Ren, J. J. Zhou, and L. He, Coulomb interaction in quasibound states of graphene quantum dots, Phys. Rev. B 101(23), 235310 (2020)

15. Y. Zhao, J. Wyrick, F. D. Natterer, J. F. RodriguezNieva, C. Lewandowski, K. Watanabe, T. Taniguchi, L. S. Levitov, N. B. Zhitenev, and J. A. Stroscio, Creating and probing electron whispering-gallery modes in graphene, Science 348(6235), 672 (2015)

16. N. M. Freitag, L. A. Chizhova, P. Nemes-Incze, C. R. Woods, R. V. Gorbachev, Y. Cao, A. K. Geim, K. S. Novoselov, J. Burgdorfer, F. Libisch, and M. Morgenstern, Electrostatically confined monolayer graphene quantum dots with orbital and valley splittings, Nano Lett. 16(9), 5798 (2016)

17. S. Y. Li, Y. Su, Y. N. Ren, and L. He, Valley polarization and inversion in strained Graphene via pseudo-Landau levels, valley splitting of real Landau levels, and confined states, Phys. Rev. Lett. 124(10), 106802 (2020)
18. L. A. Ponomarenko, F. Schedin, M. I. Katsnelson, R. Yang, E. W. Hill, K. S. Novoselov, and A. K. Geim, Chaotic Dirac billiard in graphene quantum dots, Science 320(5874), 356 (2008)

19. S. Mishra, D. Beyer, K. Eimre, J. Liu, R. Berger, O. Gröning, C. A. Pignedoli, K. Müllen, R. Fasel, X. Feng, and P. Ruffieux, Synthesis and characterization of $\pi$ extended triangulene, J. Am. Chem. Soc. 141(27), 10621 (2019)

20. S. Mishra, D. Beyer, K. Eimre, R. Ortiz, J. Fernández-Rossier, R. Berger, O. Gröning, C. A. Pignedoli, R. Fasel, X. Feng, and P. Ruffieux, Collective all-carbon magnetism in triangulene dimers, Angew. Chem. Int. Ed. 59(29), 12041 (2020)

21. J. Su, M. Telychko, P. Hu, G. Macam, P. Mutombo, H. Zhang, Y. Bao, F. Cheng, Z. Q. Huang, Z. Qiu, S. J. R. Tan, H. Lin, P. Jelínek, F. C. Chuang, J. Wu, and J. $\mathrm{Lu}$, Atomically precise bottom-up synthesis of $\pi$-extended [5]triangulene, Sci. Adv. 5(7), eaav7717 (2019)

22. N. M. Freitag, T. Reisch, L. A. Chizhova, P. Nemes-Incze, C. Holl, C. R. Woods, R. V. Gorbachev, Y. Cao, A. K. Geim, K. S. Novoselov, J. Burgdörfer, F. Libisch, and M. Morgenstern, Large tunable valley splitting in edge-free graphene quantum dots on boron nitride, Nat. Nanotechnol. 13(5), 392 (2018)

23. S.-Y. Li, Y.-N. Ren, Y.-W. Liu, M.-X. Chen, H. Jiang, and L. He, Nanoscale detection of valley-dependent spin splitting around atomic defects of graphene, 2D Mater. 6, 031005 (2019)

24. S. Jung, G. M. Rutter, N. N. Klimov, D. B. Newell, I. Calizo, A. R. Hight-Walker, N. B. Zhitenev, and J. A. Stroscio, Evolution of microscopic localization in graphene in a magnetic field from scattering resonances to quantum dots, Nat. Phys. 7(3), 245 (2011)

25. A. H. Castro Neto, F. Guinea, N. M. R. Peres, K. S. Novoselov, and A. K. Geim, The electronic properties of graphene, Rev. Mod. Phys. 81(1), 109 (2009)

26. M. Katsnelson, K. Novoselov, and A. Geim, Chiral tunnelling and the Klein paradox in graphene, Nat. Phys. 2(9), 620 (2006)

27. V. Pereira, F. Mlinar, F. M. Peeters, and P. Vasilopoulos, Confined states and direction-dependent transmission in graphene quantum wells, Phys. Rev. B 74(4), 045424 (2006)

28. M. Barbier, F. Peeters, P. Vasilopoulos, and Pereira, Dirac and Klein-Gordon particles in one-dimensional periodic potentials, Phys. Rev. B 77(11), 115446 (2008)

29. A. V. Shytov, M. S. Rudner, and L. S. Levitov, Klein backscattering and Fabry-Pérot interference in graphene heterojunctions, Phys. Rev. Lett. 101(15), 156804 (2008)

30. N. Stander, B. Huard, and D. Goldhaber-Gordon, Evidence for Klein tunneling in graphene $\mathrm{p}-\mathrm{n}$ junctions, Phys. Rev. Lett. 102(2), 026807 (2009)

31. A. F. Young and P. Kim, Quantum interference and Klein tunnelling in graphene heterojunctions, Nat. Phys. 5(3), $222(2009)$ 
32. J. H. Bardarson, M. Titov, and P. Brouwer, Electrostatic confinement of electrons in an integrable graphene quantum dot, Phys. Rev. Lett. 102(22), 226803 (2009)

33. H. Y. Chen, V. Apalkov, and T. Chakraborty, Fockdarwin states of Dirac electrons in graphene-based artificial atoms, Phys. Rev. Lett. 98(18), 186803 (2007)

34. C. Downing, D. Stone, and M. Portnoi, Zero-energy states in graphene quantum dots and rings, Phys. Rev. B 84(15), 155437 (2011)

35. A. Matulis and F. Peeters, Quasibound states of quantum dots in single and bilayer graphene, Phys. Rev. B 77(11), 115423 (2008)

36. C. Schulz, R. Heinisch, and H. Fehske, Scattering of twodimensional Dirac fermions on gate-defined oscillating quantum dots, Phys. Rev. B 91(4), 045130 (2015)

37. J. S. Wu and M. M. Fogler, Scattering of two-dimensional massless Dirac electrons by a circular potential barrier, Phys. Rev. B 90(23), 235402 (2014)

38. H. Ji, Y. Pan, and H. Liu, Evolution of quasi-bound states in the circular $\mathrm{n}-\mathrm{p}$ junction of bilayer graphene under magnetic field, Sci. Rep. 10(1), 16256 (2020)

39. Y. Pan, H. Ji, X. Q. Li, and H. Liu, Quasi-bound states in an NPN-type nanometer-scale graphene quantum dot under a magnetic field, Sci. Rep. 10(1), 20426 (2020)

40. J. Zhou, S. Cheng, W. You, and H. Jiang, Numerical study of Klein quantum dots in graphene systems, Sci. China Phys. Mech. Astron. 62(6), 67811 (2019)

41. S. M. Reimann and M. Manninen, Electronic structure of quantum dots, Rev. Mod. Phys. 74(4), 1283 (2002)

42. C. Gutiérrez, D. Walkup, F. Ghahari, C. Lewandowski, J. F. Rodriguez-Nieva, K. Watanabe, T. Taniguchi, L. S. Levitov, N. B. Zhitenev, and J. A. Stroscio, Interactiondriven quantum Hall wedding cake-like structures in graphene quantum dots, Science 361(6404), 789 (2018)

43. J. F. Rodriguez-Nieva and L. S. Levitov, Berry phase jumps and giant nonreciprocity in Dirac quantum dots, Phys. Rev. B 94(23), 235406 (2016)

44. P. McEuen, E. Foxman, J. Kinaret, U. Meirav, M. Kastner, N. S. Wingreen, and S. Wind, Self-consistent addition spectrum of a Coulomb island in the quantum Hall regime, Phys. Rev. B 45(19), 11419 (1992)

45. D. Chklovskii, B. I. Shklovskii, and L. Glazman, Electrostatics of edge channels, Phys. Rev. B 46(7), 4026 (1992)

46. M. Fogler, E. Levin, and B. Shklovskii, Chemical potential and magnetization of a Coulomb island, Phys. Rev. B 49(19), 13767 (1994)

47. Y. V. Nazarov and A. Khaetskii, Wigner molecule on the top of a quantum dot, Phys. Rev. B 49(7), 5077 (1994)

48. D. Chklovskii, K. Matveev, and B. I. Shklovskii, Ballistic conductance of interacting electrons in the quantum Hall regime, Phys. Rev. B 47(19), 12605 (1993)

49. D. Xiao, M. C. Chang, and Q. Niu, Berry phase effects on electronic properties, Rev. Mod. Phys. 82(3), 1959 (2010)

50. C. Dutreix, H. González-Herrero, I. Brihuega, M. Katsnelson, C. Chapelier, and V. Renard, Measuring the Berry phase of graphene from wavefront dislocations in Friedel oscillations, Nature 574(7777), 219 (2019)
51. K. S. Novoselov, E. McCann, S. Morozov, V. I. Fal'ko, M. Katsnelson, U. Zeitler, D. Jiang, F. Schedin, and A. Geim, Unconventional quantum Hall effect and Berry's phase of $2 \pi$ in bilayer graphene, Nat. Phys. 2(3), 177 (2006)

52. R. Resta, Macroscopic polarization in crystalline dielectrics: The geometric phase approach, Rev. Mod. Phys. 66(3), 899 (1994)

53. G. M. Rutter, S. Jung, N. N. Klimov, D. B. Newell, N. B. Zhitenev, and J. A. Stroscio, Microscopic polarization in bilayer graphene, Nat. Phys. 7(8), 649 (2011)

54. Y. Shimazaki, M. Yamamoto, I. V. Borzenets, K. Watanabe, T. Taniguchi, and S. Tarucha, Generation and detection of pure valley current by electrically induced Berry curvature in bilayer graphene, Nat. Phys. 11(12), 1032 (2015)

55. A. Varlet, M. H. Liu, V. Krueckl, D. Bischoff, P. Simonet, K. Watanabe, T. Taniguchi, K. Richter, K. Ensslin, and T. Ihn, Fabry-Pérot interference in gapped bilayer graphene with broken anti-Klein tunneling, Phys. Rev. Lett. 113(11), 116601 (2014)

56. Y. Zhang, Y. W. Tan, H. L. Stormer, and P. Kim, Experimental observation of the quantum Hall effect and Berry's phase in graphene, Nature 438(7065), 201 (2005)

57. K. S. Novoselov, A. K. Geim, S. V. Morozov, D. Jiang, M. I. Katsnelson, I. V. Grigorieva, S. V. Dubonos, and A. A. Firsov, Two-dimensional gas of massless Dirac fermions in graphene, Nature 438(7065), 197 (2005)

58. D. L. Miller, K. D. Kubista, G. M. Rutter, M. Ruan, W. A. de Heer, P. N. First, and J. A. Stroscio, Observing the quantization of zero mass carriers in graphene, Science 324(5929), 924 (2009)

59. Z. Q. Fu, Y. Zhang, J. B. Qiao, D. L. Ma, H. Liu, Z. H. Guo, Y. C. Wei, J. Y. Hu, Q. Xiao, X. R. Mao, and L. He, Spatial confinement, magnetic localization, and their interactions on massless Dirac fermions, Phys. Rev. B 98(24), 241401 (2018)

60. A. Filinov, M. Bonitz, and Y. E. Lozovik, Wigner crystallization in mesoscopic 2D electron systems, Phys. Rev. Lett. 86(17), 3851 (2001)

61. C. H. Zhang and Y. N. Joglekar, Wigner crystal and bubble phases in graphene in the quantum Hall regime, Phys. Rev. B 75(24), 245414 (2007)

62. K. A. Guerrero-Becerra and M. Rontani, Wigner localization in a graphene quantum dot with a mass gap, Phys. Rev. B 90(12), 125446 (2014)

63. H. González-Herrero, J. M. Gomez-Rodriguez, P. Mallet, M. Moaied, J. J. Palacios, C. Salgado, M. M. Ugeda, J. Y. Veuillen, F. Yndurain, and I. Brihuega, Atomic-scale control of graphene magnetism by using hydrogen atoms, Science 352(6284), 437 (2016)

64. Y. Zhang, S. Y. Li, H. Huang, W. T. Li, J. B. Qiao, W. X. Wang, L. J. Yin, K. K. Bai, W. Duan, and L. He, Scanning tunneling microscopy of the $\pi$ magnetism of a single carbon vacancy in graphene, Phys. Rev. Lett. 117(16), 166801 (2016) 
65. Y. Cao, V. Fatemi, A. Demir, S. Fang, S. L. Tomarken, J. Y. Luo, J. D. Sanchez-Yamagishi, K. Watanabe, T. Taniguchi, E. Kaxiras, R. C. Ashoori, and P. JarilloHerrero, Correlated insulator behaviour at half-filling in magic-angle graphene superlattices, Nature 556(7699), 80 (2018)

66. Y. Cao, V. Fatemi, S. Fang, K. Watanabe, T. Taniguchi, E. Kaxiras, and P. Jarillo-Herrero, Unconventional superconductivity in magic-angle graphene superlattices, $\mathrm{Na}$ ture 556(7699), 43 (2018)

67. Y. W. Liu, J. B. Qiao, C. Yan, Y. Zhang, S. Y. Li, and L. He, Magnetism near half-filling of a van Hove singularity in twisted graphene bilayer, Phys. Rev. B 99, 201408(R) (2019)

68. G. Chen, A. L. Sharpe, P. Gallagher, I. T. Rosen, E. J. Fox, L. Jiang, B. Lyu, H. Li, K. Watanabe, T. Taniguchi, J. Jung, Z. Shi, D. Goldhaber-Gordon, Y. Zhang, and F. Wang, Signatures of tunable superconductivity in a trilayer graphene moiré superlattice, Nature 572(7768), 215 (2019)

69. A. L. Sharpe, E. J. Fox, A. W. Barnard, J. Finney, K. Watanabe, T. Taniguchi, M. A. Kastner, and D. Goldhaber-Gordon, Emergent ferromagnetism near three-quarters filling in twisted bilayer graphene, Science 365(6453), 605 (2019)

70. A. F. Young, C. R. Dean, L. Wang, H. Ren, P. CaddenZimansky, K. Watanabe, T. Taniguchi, J. Hone, K. L. Shepard, and P. Kim, Spin and valley quantum Hall ferromagnetism in graphene, Nat. Phys. 8(7), 550 (2012)

71. S. Y. Li, Y. Zhang, L. J. Yin, and L. He, Scanning tunneling microscope study of quantum Hall isospin ferromagnetic states in the zero Landau level in a graphene monolayer, Phys. Rev. B 100(8), 085437 (2019)

72. G. Chen, L. Jiang, S. Wu, B. Lyu, H. Li, B. L. Chittari, K. Watanabe, T. Taniguchi, Z. Shi, J. Jung, Y. Zhang, and F. Wang, Evidence of a gate-tunable Mott insulator in a trilayer graphene moiré superlattice, Nat. Phys. 15(3), 237 (2019)

73. L. J. Yin, L. J. Shi, S. Y. Li, Y. Zhang, Z. H. Guo, and L. He, High-magnetic-field tunneling spectra of ABCstacked trilayer graphene on graphite, Phys. Rev. Lett. 122(14), 146802 (2019)

74. G. Bester, D. Reuter, L. He, A. Zunger, P. Kailuweit, A. Wieck, U. Zeitler, J. Maan, O. Wibbelhoff, and A. Lorke, Experimental imaging and atomistic modeling of electron and hole quasiparticle wave functions in In AsGaAs quantum dots, Phys. Rev. B 76(7), 075338 (2007)

75. F. Cavaliere, U. D. Giovannini, M. Sassetti, and B. Kramer, Transport properties of quantum dots in the Wigner molecule regime, New J. Phys. 11(12), 123004 (2009)

76. A. Ghosal, A. Güçlü, C. Umrigar, D. Ullmo, and H. U. Baranger, Correlation-induced inhomogeneity in circular quantum dots, Nat. Phys. 2(5), 336 (2006)

77. A. Güçlü, P. Potasz, O. Voznyy, M. Korkusinski, and P. Hawrylak, Magnetism and correlations in fractionally filled degenerate shells of graphene quantum dots, Phys. Rev. Lett. 103(24), 246805 (2009)
78. P. Potasz, A. Güçlü, and P. Hawrylak, Spin and electronic correlations in gated graphene quantum rings, Phys. Rev. B 82(7), 075425 (2010)

79. M. Rontani and E. Molinari, Imaging quasiparticle wave functions in quantum dots via tunneling spectroscopy, Phys. Rev. B 71(23), 233106 (2005)

80. B. Wunsch, T. Stauber, and F. Guinea, Electron-electron interactions and charging effects in graphene quantum dots, Phys. Rev. B 77(3), 035316 (2008)

81. A. Güçlü, P. Potasz, and P. Hawrylak, Excitonic absorption in gate-controlled graphene quantum dots, Phys. Rev. B 82(15), 155445 (2010)

82. Y. Li, H. Shu, S. Wang, and J. Wang, Electronic and optical properties of graphene quantum dots: The role of many-body effects, J. Phys. Chem. C 119(9), 4983 (2015)

83. A. Franceschetti and A. Zunger, Direct pseudopotential calculation of exciton Coulomb and exchange energies in semiconductor quantum dots, Phys. Rev. Lett. 78(5), 915 (1997)

84. A. Bostwick, F. Speck, T. Seyller, K. Horn, M. Polini, R. Asgari, A. H. MacDonald, and E. Rotenberg, Observation of plasmarons in quasi-freestanding doped graphene, Science 328(5981), 999 (2010)

85. D. Elias, R. V. Gorbachev, A. Mayorov, S. Morozov, A. Zhukov, P. Blake, L. Ponomarenko, I. V. Grigorieva, K. S. Novoselov, F. Guinea, and A. K. Geim, Dirac cones reshaped by interaction effects in suspended graphene, Nat. Phys. 7(9), 701 (2011)

86. J. P. Reed, B. Uchoa, Y. I. Joe, Y. Gan, D. Casa, E. Fradkin, and P. Abbamonte, The effective fine-structure constant of freestanding graphene measured in graphite, Science 330(6005), 805 (2010)

87. D. A. Siegel, C. H. Park, C. Hwang, J. Deslippe, A. V. Fedorov, S. G. Louie, and A. Lanzara, Many-body interactions in quasi-freestanding graphene, Proc. Natl. Acad. Sci. USA 108(28), 11365 (2011)

88. Y. Wang, V. W. Brar, A. V. Shytov, Q. Wu, W. Regan, H. Z. Tsai, A. Zettl, L. S. Levitov, and M. F. Crommie, Mapping Dirac quasiparticles near a single Coulomb impurity on graphene, Nat. Phys. 8(9), 653 (2012)

89. R. H. Blick, D. Pfannkuche, R. Haug, K. Klitzing, and K. Eberl, Formation of a coherent mode in a double quantum dot, Phys. Rev. Lett. 80(18), 4032 (1998)

90. D. Dixon, L. Kouwenhoven, P. McEuen, Y. Nagamune, J. Motohisa, and H. Sakaki, Influence of energy level alignment on tunneling between coupled quantum dots, Phys. Rev. B 53(19), 12625 (1996)

91. A. W. Holleitner, R. H. Blick, A. K. Hüttel, K. Eberl, and J. P. Kotthaus, Probing and Controlling the Bonds of an Artificial Molecule, Science 297(5578), 70 (2002)

92. T. Oosterkamp, T. Fujisawa, W. Van Der Wiel, K. Ishibashi, R. Hijman, S. Tarucha, and L. P. Kouwenhoven, Microwave spectroscopy of a quantum-dot molecule, Nature 395(6705), 873 (1998)

93. B. Partoens and F. Peeters, Molecule-type phases and Hund's rule in vertically coupled quantum dots, Phys. Rev. Lett. 84(19), 4433 (2000) 
94. G. Schedelbeck, W. Wegscheider, M. Bichler, and G. Abstreiter, Coupled quantum dots fabricated by cleaved edge overgrowth: From artificial atoms to molecules, Science 278(5344), 1792 (1997)

95. F. Waugh, M. Berry, C. Crouch, C. Livermore, D. Mar, R. Westervelt, K. Campman, and A. Gossard, Measuring interactions between tunnel-coupled quantum dots, Phys. Rev. B 53(3), 1413 (1996)

96. A. P. Alivisatos, Semiconductor clusters, nanocrystals, and quantum dots, Science 271(5251), 933 (1996)

97. R. E. Bailey and S. Nie, Alloyed semiconductor quantum dots: Tuning the optical properties without changing the particle size, J. Am. Chem. Soc. 125(23), 7100 (2003)

98. L. Banszerus, B. Frohn, A. Epping, D. Neumaier, K. Watanabe, T. Taniguchi, and C. Stampfer, Gate-defined electron-hole double dots in bilayer graphene, Nano Lett. 18(8), 4785 (2018)

99. M. Bayer, P. Hawrylak, K. Hinzer, S. Fafard, M. Korkusinski, Z. Wasilewski, O. Stern, and A. Forchel, Coupling and entangling of quantum states in quantum dot molecules, Science 291(5503), 451 (2001)

100. I. L. Chuang, L. M. Vandersypen, X. Zhou, D. W. Leung, and S. Lloyd, Experimental realization of a quantum algorithm, Nature 393(6681), 143 (1998)

101. N. Craig, J. Taylor, E. Lester, C. Marcus, M. Hanson, and A. Gossard, Tunable nonlocal spin control in a coupledquantum dot system, Science 304(5670), 565 (2004)

102. M. Doty, M. Scheibner, I. Ponomarev, E. Stinaff, A. Bracker, V. Korenev, T. Reinecke, and D. Gammon, Electrically tunable $\mathrm{g}$ factors in quantum dot molecular spin states, Phys. Rev. Lett. 97(19), 197202 (2006)

103. T. Fujisawa, T. H. Oosterkamp, W. G. Van der Wiel, B. W. Broer, R. Aguado, S. Tarucha, and L. P. Kouwenhoven, Spontaneous emission spectrum in double quantum dot devices, Science 282(5390), 932 (1998)

104. C. R. Kagan and C. B. Murray, Charge transport in strongly coupled quantum dot solids, Nat. Nanotechnol. 10(12), 1013 (2015)

105. J. Lobo-Checa, M. Matena, K. Müller, J. H. Dil, F. Meier, L. H. Gade, T. A. Jung, and M. Stöhr, Band formation from coupled quantum dots formed by a nanoporous network on a Copper surface, Science 325(5938), 300 (2009)

106. Y. Pan, J. Yang, S. C. Erwin, K. Kanisawa, and S. Fölsch, Reconfigurable quantum-dot molecules created by atom manipulation, Phys. Rev. Lett. 115(7), 076803 (2015)

107. L. Robledo, J. Elzerman, G. Jundt, M. Atatüre, A. Högele, S. Fält, and A. Imamoglu, Conditional dynamics of interacting quantum dots, Science 320(5877), 772 (2008)

108. M. Scheibner, M. Yakes, A. S. Bracker, I. V. Ponomarev, M. Doty, C. Hellberg, L. Whitman, T. Reinecke, and D. Gammon, Optically mapping the electronic structure of coupled quantum dots, Nat. Phys. 4(4), 291 (2008)

109. E. A. Stinaff, M. Scheibner, A. S. Bracker, I. V. Ponomarev, V. L. Korenev, M. E. Ware, M. F. Doty, T. L. Reinecke, and D. Gammon, Optical signatures of coupled quantum dots, Science 311(5761), 636 (2006)
110. T. Unold, K. Mueller, C. Lienau, T. Elsaesser, and A. D. Wieck, Optical control of excitons in a pair of quantum dots coupled by the dipole-dipole interaction, Phys. Rev. Lett. 94(13), 137404 (2005)

111. W. G. van der Wiel, S. De Franceschi, J. M. Elzerman, T. Fujisawa, S. Tarucha, and L. P. Kouwenhoven, Electron transport through double quantum dots, Rev. Mod. Phys. 75(1), 1 (2002)

112. A. D. Yoffe, Semiconductor quantum dots and related systems: Electronic, optical, luminescence and related properties of low dimensional systems, Adv. Phys. 50(1), 1 (2001)

113. Z. Q. Fu, Y. Pan, J. J. Zhou, K. K. Bai, D. L. Ma, Y. Zhang, J. B. Qiao, H. Jiang, H. Liu, and L. He, Relativistic artificial molecules realized by two coupled graphene quantum dots, Nano Lett. 20(9), 6738 (2020)

114. J. B. Qiao, H. Jiang, H. Liu, H. Yang, N. Yang, K. Y. Qiao, and L. He, Bound states in nanoscale graphene quantum dots in a continuous graphene sheet, Phys. Rev. B 95(8), 081409 (2017)

115. J. Güttinger, C. Stampfer, S. Hellmüller, F. Molitor, T. Ihn, and K. Ensslin, Charge detection in graphene quantum dots, Appl. Phys. Lett. 93(21), 212102 (2008)

116. X. L. Liu, D. Hug, and L. M. Vandersypen, Gate-defined graphene double quantum dot and excited state spectroscopy, Nano Lett. 10(5), 1623 (2010)

117. F. Molitor, S. Dröscher, J. Güttinger, A. Jacobsen, C. Stampfer, T. Ihn, and K. Ensslin, Transport through graphene double dots, Appl. Phys. Lett. 94(22), 222107 (2009)

118. P. Silvestrov and K. Efetov, Quantum dots in graphene, Phys. Rev. Lett. 98(1), 016802 (2007)

119. C. Stampfer, J. Güttinger, F. Molitor, D. Graf, T. Ihn, and K. Ensslin, Tunable Coulomb blockade in nanostructured graphene, Appl. Phys. Lett. 92(1), 012102 (2008)

120. A. C. Bleszynski, F. A. Zwanenburg, R. Westervelt, A. L. Roest, E. P. Bakkers, and L. P. Kouwenhoven, Scanned probe imaging of quantum dots inside InAs nanowires, Nano Lett. 7(9), 2559 (2007)

121. A. Deshpande, W. Bao, Z. Zhao, C. Lau, and B. J. LeRoy, Imaging charge density fluctuations in graphene using Coulomb blockade spectroscopy, Phys. Rev. B 83(15), 155409 (2011)

122. P. Fallahi, A. C. Bleszynski, R. M. Westervelt, J. Huang, J. D. Walls, E. J. Heller, M. Hanson, and A. C. Gossard, Imaging a single-electron quantum dot, Nano Lett. 5(2), $223(2005)$

123. K. A. Ritter and J. W. Lyding, The influence of edge structure on the electronic properties of graphene quantum dots and nanoribbons, Nat. Mater. 8(3), 235 (2009)

124. X. Wang, Y. Ouyang, L. Jiao, H. Wang, L. Xie, J. Wu, J. Guo, and H. Dai, Graphene nanoribbons with smooth edges behave as quantum wires, Nat. Nanotechnol. 6(9), $563(2011)$

125. M. T. Woodside and P. L. McEuen, Scanned probe imaging of single-electron charge states in nanotube quantum dots, Science 296(5570), 1098 (2002) 
126. D. Walkup, F. Ghahari, C. Gutiérrez, K. Watanabe, T. Taniguchi, N. B. Zhitenev, and J. A. Stroscio, Tuning single-electron charging and interactions between compressible Landau level islands in graphene, Phys. Rev. B 101(3), 035428 (2020)

127. M. Amman, R. Wilkins, E. Ben-Jacob, P. Maker, and R. Jaklevic, Analytic solution for the current-voltage characteristic of two mesoscopic tunnel junctions coupled in series, Phys. Rev. B 43(1), 1146 (1991)

128. A. Hanna, and M. Tinkham, Variation of the Coulomb staircase in a two-junction system by fractional electron charge, Phys. Rev. B 44(11), 5919 (1991)

129. V. W. Brar, R. Decker, H. M. Solowan, Y. Wang, L. Maserati, K. T. Chan, H. Lee, Ç. O. Girit, A. Zettl, S. G. Louie, M. L. Cohen, and M. F. Crommie, Gate-controlled ionization and screening of cobalt adatoms on a graphene surface, Nat. Phys. 7(1), 43 (2011)

130. D. Wong, L. Jr Velasco, J. Ju, S. Lee, H. Z. Kahn, C. Tsai, T. Germany, K. Taniguchi, A. Watanabe, F. Zettl, Wang, and M. F. Crommie, Characterization and manipulation of individual defects in insulating hexagonal boron nitride using scanning tunnelling microscopy, Nat. Nanotechnol. 10(11), 949 (2015)

131. Y. J. Song, A. F. Otte, Y. Kuk, Y. Hu, D. B. Torrance, P. N. First, W. A. de Heer, H. Min, S. Adam, M. D. Stiles, A. H. MacDonald, and J. A. Stroscio, High-resolution tunnelling spectroscopy of a graphene quartet, Nature 467(7312), 185 (2010)

132. L. J. Yin, S. Y. Li, J. B. Qiao, J. C. Nie, and L. He, Landau quantization in graphene monolayer, Bernal bilayer, and Bernal trilayer on graphite surface, Phys. Rev. B 91(11), 115405 (2015)

133. S. Y. Li, H. Liu, J. B. Qiao, H. Jiang, and L. He, Magnetic-field-controlled negative differential conductance in scanning tunneling spectroscopy of graphene np-n junction resonators, Phys. Rev. B 97(11), 115442 (2018)

134. S. Y. Li, K. K. Bai, W. J. Zuo, Y. W. Liu, Z. Q. Fu, W. X. Wang, Y. Zhang, L. J. Yin, J. B. Qiao, and L. He, Tunneling spectra of a quasifreestanding graphene monolayer, Phys. Rev. Appl. 9(5), 054031 (2018)

135. T. Low and F. Guinea, Strain-induced pseudomagnetic field for novel graphene electronics, Nano Lett. 10(9), 3551 (2010)

136. D. Abanin and D. Pesin, Interaction-induced topological insulator states in strained graphene, Phys. Rev. Lett. 109(6), 066802 (2012)

137. B. Uchoa and Y. Barlas, Superconducting states in pseudo-Landau-levels of strained graphene, Phys. Rev. Lett. 111(4), 046604 (2013)

138. B. Roy, Z. X. Hu, and K. Yang, Theory of unconventional quantum Hall effect in strained graphene, Phys. Rev. B 87(12), 121408 (2013)

139. D. B. Zhang, G. Seifert, and K. Chang, Strain-induced pseudomagnetic fields in twisted graphene nanoribbons, Phys. Rev. Lett. 112(9), 096805 (2014)

140. G. Wakker, R. P. Tiwari, and M. Blaauboer, Localization and circulating currents in curved graphene devices, Phys. Rev. B 84(19), 195427 (2011)
141. B. Amorim, A. Cortijo, F. De Juan, A. Grushin, F. Guinea, A. Gutiérrez-Rubio, H. Ochoa, V. Parente, R. Roldán, P. San-Jose, J. Schiefele, M. Sturla, and M. A. H. Vozmediano, Novel effects of strains in graphene and other two dimensional materials, Phys. Rep. 617, 1 (2016)

142. M. Settnes, S. R. Power, and A. P. Jauho, Pseudomagnetic fields and triaxial strain in graphene, Phys. Rev. B 93(3), 035456 (2016)

143. S. Y. Li, Y. Su, Y. N. Ren, and L. He, Valley polarization and inversion in strained graphene via pseudoLandau levels, valley splitting of real Landau levels, and confined states, Phys. Rev. Lett. 124, 106802 (2020)

144. E. McCann, Asymmetry gap in the electronic band structure of bilayer graphene, Phys. Rev. B 74(16), 161403 (2006)

145. Y. Zhang, T. T. Tang, C. Girit, Z. Hao, M. C. Martin, A. Zettl, M. F. Crommie, Y. R. Shen, and F. Wang, Direct observation of a widely tunable bandgap in bilayer graphene, Nature 459(7248), 820 (2009)

146. J. B. Oostinga, H. B. Heersche, X. Liu, A. F. Morpurgo, and L. M. Vandersypen, Gate-induced insulating state in bilayer graphene devices, Nat. Mater. 7(2), 151 (2008)

147. L. J. Yin, H. Jiang, J. B. Qiao, and L. He, Direct imaging of topological edge states at a bilayer graphene domain wall, Nat. Commun. 7(1), 11760 (2016)

148. L. Ju, Z. Shi, N. Nair, Y. Lv, C. Jin, C. Jr Velasco, H. A. Ojeda-Aristizabal, M. C. Bechtel, A. Martin, J. Zettl, Analytis, and F. Wang, Topological valley transport at bilayer graphene domain walls, Nature 520(7549), 650 (2015)

149. L. J. Yin, Y. Zhang, J. B. Qiao, S. Y. Li, and L. He, Experimental observation of surface states and Landau levels bending in bilayer graphene, Phys. Rev. B 93(12), $125422(2016)$

150. Y. T. Zhang, X. Xie, and Q. Sun, Effect of Zeeman splitting and interlayer bias potential on electron transport in bilayer graphene, Phys. Rev. B 86(3), 035447 (2012)

151. J. Li, K. Wang, K. J. McFaul, Z. Zern, Y. Ren, K. Watanabe, T. Taniguchi, Z. Qiao, and J. Zhu, Gate-controlled topological conducting channels in bilayer graphene, Nat. Nanotechnol. 11(12), 1060 (2016)

152. M. T. Allen, J. Martin, and A. Yacoby, Gate-defined quantum confinement in suspended bilayer graphene, Nat. Commun. 3(1), 934 (2012)

153. A. M. Goossens, S. C. Driessen, T. A. Baart, K. Watanabe, T. Taniguchi, and L. M. Vandersypen, Gate-defined confinement in bilayer graphene-hexagonal boron nitride hybrid devices, Nano Lett. 12(9), 4656 (2012)

154. J. Jr Velasco, D. Lee, S. Wong, H. Z. Kahn, J. Tsai, T. Costello, T. Umeda, K. Taniguchi, Watanabe, A. Zettl, F. Wang, and M. F. Crommie, Visualization and control of single-electron charging in bilayer graphene quantum dots, Nano Lett. 18(8), 5104 (2018)

155. Y. Lee, A. Knothe, H. Overweg, M. Eich, C. Gold, A. Kurzmann, V. Klasovika, T. Taniguchi, K. Wantanabe, V. Fal'ko, T. Ihn, K. Ensslin, and P. Rickhaus, Tunable valley splitting due to topological orbital magnetic moment in bilayer graphene quantum point contacts, Phys. Rev. Lett. 124(12), 126802 (2020) 
156. M. Eich, F. Herman, R. Pisoni, H. Overweg, A. Kurzmann, Y. Lee, P. Rickhaus, K. Watanabe, T. Taniguchi, M. Sigrist, T. Ihn, and K. Ensslin, Spin and valley states in gate-defined bilayer graphene quantum dots, Phys. Rev. X 8(3), 031023 (2018)

157. A. Kurzmann, M. Eich, H. Overweg, M. Mangold, F. Herman, P. Rickhaus, R. Pisoni, Y. Lee, R. Garreis, C. Tong, K. Watanabe, T. Taniguchi, K. Ensslin, and T. Ihn, Excited states in bilayer graphene quantum dots, Phys. Rev. Lett. 123(2), 026803 (2019)

158. N. Gu, M. Rudner, and L. Levitov, Chirality-assisted electronic cloaking of confined states in bilayer graphene, Phys. Rev. Lett. 107(15), 156603 (2011)

159. Z. Ge, F. Joucken, E. Quezada, D. R. Da Costa, J. Davenport, B. Giraldo, T. Taniguchi, K. Watanabe, N. P. Kobayashi, T. Low, and J. Jr Velasco, Visualization and manipulation of bilayer graphene quantum dots with broken rotational symmetry and nontrivial topology, Nano Lett. 20(12), 8682 (2020)

160. C. H. Park and N. Marzari, Berry phase and pseudospin winding number in bilayer graphene, Phys. Rev. B 84(20), 205440 (2011)

161. K. F. Mak, C. H. Lui, J. Shan, and T. F. Heinz, Observation of an electric-field-induced band gap in bilayer graphene by infrared spectroscopy, Phys. Rev. Lett. 102(25), 256405 (2009)

162. T. Ohta, A. Bostwick, T. Seyller, K. Horn, and E. Rotenberg, Controlling the electronic structure of bilayer graphene, Science 313(5789), 951 (2006)

163. Z. Hou, Y. F. Zhou, X. Xie, and Q. F. Sun, Berry phase induced valley level crossing in bilayer graphene quantum dots, Phys. Rev. B 99(12), 125422 (2019)

164. R. Du, M. H. Liu, J. Mohrmann, F. Wu, R. Krupke, H. Von Löhneysen, K. Richter, and R. Danneau, Tuning anti-Klein to Klein tunneling in bilayer graphene, Phys. Rev. Lett. 121(12), 127706 (2018)

165. W. Yao, D. Xiao, and Q. Niu, Valley-dependent optoelectronics from inversion symmetry breaking, Phys. Rev. B $77(23), 235406$ (2008)

166. R. Gorbachev, J. Song, G. Yu, A. Kretinin, F. Withers, Y. Cao, A. Mishchenko, I. Grigorieva, K. S. Novoselov, L. Levitov, and A. K. Geim, Detecting topological currents in graphene superlattices, Science 346(6208), 448 (2014)

167. K. F. Mak, K. L. McGill, J. Park, and P. L. McEuen, The valley Hall effect in $\mathrm{MoS}_{2}$ transistors, Science 344(6191), 1489 (2014)

168. S. Y. Xu, Q. Ma, H. Shen, V. Fatemi, S. Wu, T. R. Chang, G. Chang, A. M. M. Valdivia, C. K. Chan, Q. D. Gibson, J. Zhou, Z. Liu, K. Watanabe, T. Taniguchi, H. Lin, R. J. Cava, L. Fu, N. Gedik, and P. Jarillo-Herrero, Electrically switchable Berry curvature dipole in the monolayer topological insulator $\mathrm{WTe}_{2}$, Nat. Phys. 14(9), 900 (2018)

169. H. Weng, C. Fang, Z. Fang, B. A. Bernevig, and X. Dai, Weyl semimetal phase in noncentrosymmetric transitionmetal monophosphides, Phys. Rev. X 5(1), 011029 (2015)
170. S. Y. Xu, I. Belopolski, N. Alidoust, M. Neupane, G. Bian, C. Zhang, R. Sankar, G. Chang, Z. Yuan, C. C. Lee, S. M. Huang, H. Zheng, J. Ma, D. S. Sanchez, B. K. Wang, A. Bansil, F. Chou, P. P. Shibayev, H. Lin, S. Jia, and M. Z. Hasan, Discovery of a Weyl fermion semimetal and topological Fermi arcs, Science 349(6248), 613 (2015)

171. B. Lv, H. Weng, B. Fu, X. P. Wang, H. Miao, J. Ma, P. Richard, X. Huang, L. Zhao, G. Chen, Z. Fang, X. Dai, T. Qian, and H. Ding, Experimental discovery of Weyl semimetal TaAs, Phys. Rev. X 5(3), 031013 (2015)

172. M. Eich, R. Pisoni, A. Pally, H. Overweg, A. Kurzmann, Y. Lee, P. Rickhaus, K. Watanabe, T. Taniguchi, K. Ensslin, and T. Ihn, Coupled quantum dots in bilayer graphene, Nano Lett. 18(8), 5042 (2018)

173. L. Banszerus, S. Möller, E. Icking, K. Watanabe, T. Taniguchi, C. Volk, and C. Stampfer, Single-electron double quantum dots in bilayer graphene, Nano Lett. 20(3), 2005 (2020)

174. E. Clar and D. Stewart, Aromatic hydrocarbons. LXV. Triangulene derivatives, J. Am. Chem. Soc. 75(11), 2667 (1953)

175. M. Melle-Franco, When $1+1$ is odd, Nat. Nanotechnol. 12(4), 292 (2017)

176. Y. Morita, S. Suzuki, K. Sato, and T. Takui, Synthetic organic spin chemistry for structurally well-defined openshell graphene fragments, Nat. Chem. 3(3), 197 (2011)

177. E. H. Lieb, Two theorems on the Hubbard model, Phys. Rev. Lett. 62(10), 1201 (1989)

178. P. Potasz, A. Güçlü, and P. Hawrylak, Zero-energy states in triangular and trapezoidal graphene structures, Phys. Rev. B 81(3), 033403 (2010)

179. J. Fernández-Rossier and J. J. Palacios, Magnetism in graphene nanoislands, Phys. Rev. Lett. 99(17), 177204 (2007)

180. W. L. Wang, S. Meng, and E. Kaxiras, Graphene nanoflakes with large spin, Nano Lett. 8(1), 241 (2008)

181. A. Heinrich, J. Gupta, C. Lutz, and D. Eigler, Singleatom spin-flip spectroscopy, Science 306(5695), 466 (2004)

182. C. F. Hirjibehedin, C. P. Lutz, and A. J. Heinrich, Spin coupling in engineered atomic structures, Science 312(5776), 1021 (2006)

183. S. Baumann, W. Paul, T. Choi, C. P. Lutz, A. Ardavan, and A. J. Heinrich, Electron paramagnetic resonance of individual atoms on a surface, Science 350(6259), 417 (2015)

184. W. L. Wang, O. V. Yazyev, S. Meng, and E. Kaxiras, Topological frustration in graphene nanoflakes: Magnetic order and spin logic devices, Phys. Rev. Lett. 102(15), 157201 (2009)

185. W. Han, R. K. Kawakami, M. Gmitra, and J. Fabian, Graphene spintronics, Nat. Nanotechnol. 9(10), 794 (2014)

186. N. Pavliček, A. Mistry, Z. Majzik, N. Moll, G. Meyer, D. J. Fox, and L. Gross, Synthesis and characterization of triangulene, Nat. Nanotechnol. 12(4), 308 (2017) 
187. G. Allinson, R. J. Bushby, J. L. Paillaud, D. Oduwole, and K. Sales, ESR spectrum of a stable triplet $\pi$ biradical: Trioxytriangulene, J. Am. Chem. Soc. 115(5), 2062 (1993)

188. J. Inoue, K. Fukui, T. Kubo, S. Nakazawa, K. Sato, D. Shiomi, Y. Morita, K. Yamamoto, T. Takui, and K. Nakasuji, The first detection of a Clar's hydrocarbon, 2,6,10-tri-tert-butyltriangulene: A ground-state triplet of non-Kekulé polynuclear benzenoid hydrocarbon, J. Am. Chem. Soc. 123(50), 12702 (2001)

189. G. Allinson, R. J. Bushby, M. V. Jesudason, J.-L. Paillaud, and N. Taylor, The synthesis of singlet ground state derivatives of non-Kekulé polynuclear aromatics, $J$. Chem. Soc. Perkin Trans. 2, 147 (1997)

190. N. Pavliček, I. Swart, J. Niedenführ, G. Meyer, and J. Repp, Symmetry dependence of vibration-assisted tunneling, Phys. Rev. Lett. 110(13), 136101 (2013)

191. J. Repp, G. Meyer, S. Paavilainen, F. E. Olsson, and M. Persson, Imaging bond formation between a gold atom and pentacene on an insulating surface, Science 312(5777), 1196 (2006)

192. P. Jia, W. Chen, J. Qiao, M. Zhang, X. Zheng, Z. Xue, R. Liang, C. Tian, L. He, Z. Di, and X. Wang, Programmable graphene nanobubbles with three-fold symmetric pseudo-magnetic fields, Nat. Commun. 10(1), 3127 (2019)

193. Y. W. Liu, Y. Su, X. F. Zhou, L. J. Yin, C. Yan, S. Y. Li, W. Yan, S. Han, Z. Q. Fu, Y. Zhang, Q. Yang, Y. N. Ren, and L. He, Tunable lattice reconstruction, triangular network of chiral one-dimensional states, and bandwidth of flat bands in magic angle twisted bilayer graphene, Phys. Rev. Lett. 125(23), 236102 (2020)

194. S. Y. Li, Y. Zhang, Y. N. Ren, J. Liu, X. Dai, and L. He, Experimental evidence for orbital magnetic moments generated by moiré-scale current loops in twisted bilayer graphene, Phys. Rev. B 102(12), 121406 (2020)

195. J. Cai, P. Ruffieux, R. Jaafar, M. Bieri, T. Braun, S. Blankenburg, M. Muoth, A. P. Seitsonen, M. Saleh, X. Feng, K. Müllen, and R. Fasel, Atomically precise bottom-up fabrication of graphene nanoribbons, Nature 466(7305), 470 (2010)

196. P. Ruffieux, S. Wang, B. Yang, C. Sánchez-Sánchez, J. Liu, T. Dienel, L. Talirz, P. Shinde, C. A. Pignedoli, D. Passerone, T. Dumslaff, X. Feng, K. Müllen, and R. Fasel, On-surface synthesis of graphene nanoribbons with zigzag edge topology, Nature 531(7595), 489 (2016)

197. M. Di Giovannantonio, O. Deniz, J. I. Urgel, R. Widmer, T. Dienel, S. Stolz, C. Sánchez-Sánchez, M. Muntwiler, T. Dumslaff, R. Berger, A. Narita, X. Feng, K. Müllen, P. Ruffieux, and R. Fasel, On-surface growth dynamics of graphene nanoribbons: The role of halogen functionalization, ACS Nano 12(1), 74 (2018)

198. Y. C. Chen, T. Cao, C. Chen, Z. Pedramrazi, D. Haberer, D. G. De Oteyza, F. R. Fischer, S. G. Louie, and M. F. Crommie, Molecular bandgap engineering of bottom-up synthesized graphene nanoribbon heterojunctions, Nat. Nanotechnol. 10(2), 156 (2015)
199. M. Treier, C. A. Pignedoli, T. Laino, R. Rieger, K. Müllen, D. Passerone, and R. Fasel, Surface-assisted cyclodehydrogenation provides a synthetic route towards easily processable and chemically tailored nanographenes, Nat. Chem. 3(1), 61 (2011)

200. J. Cai, C. A. Pignedoli, L. Talirz, P. Ruffieux, H. Söde, L. Liang, V. Meunier, R. Berger, R. Li, X. Feng, K. Müllen, and R. Fasel, Graphene nanoribbon heterojunctions, Nat. Nanotechnol. 9(11), 896 (2014)

201. O. Gröning, S. Wang, X. Yao, C. A. Pignedoli, G. Borin Barin, C. Daniels, A. Cupo, V. Meunier, X. Feng, A. Narita, K. Müllen, P. Ruffieux, and R. Fasel, Engineering of robust topological quantum phases in graphene nanoribbons, Nature 560(7717), 209 (2018)

202. S. Wang, N. Kharche, E. Costa Girão, X. Feng, K. Müllen, V. Meunier, R. Fasel, and P. Ruffieux, Quantum dots in graphene nanoribbons, Nano Lett. 17(7), 4277 (2017)

203. F. J. Giessibl, Advances in atomic force microscopy, Rev. Mod. Phys. 75(3), 949 (2003)

204. F. Mohn, B. Schuler, L. Gross, and G. Meyer, Different tips for high-resolution atomic force microscopy and scanning tunneling microscopy of single molecules, Appl. Phys. Lett. 102(7), 073109 (2013)

205. L. Gross, F. Mohn, N. Moll, P. Liljeroth, and G. Meyer, The chemical structure of a molecule resolved by atomic force microscopy, Science 325(5944), 1110 (2009)

206. F. J. Giessibl, High-speed force sensor for force microscopy and profilometry utilizing a quartz tuning fork, Appl. Phys. Lett. 73(26), 3956 (1998)

207. D. J. Choi, N. Lorente, J. Wiebe, K. Von Bergmann, A. F. Otte, and A. J. Heinrich, Atomic spin chains on surfaces, Rev. Mod. Phys. 91(4), 041001 (2019)

208. G. Trinquier, N. Suaud, N. Guihéry, and J. P. Malrieu, Designing magnetic organic lattices from high-spin polycyclic units, ChemPhysChem 12(16), 3020 (2011)

209. X. Li, J. Zhou, Q. Wang, X. Chen, Y. Kawazoe, and P. Jena, Magnetism of two-dimensional triangular nanoflake-based kagome lattices, New J. Phys. 14(3), $033043(2012)$

210. X. Li and Q. Wang, Tunable ferromagnetism in assembled two dimensional triangular graphene nanoflakes, Phys. Chem. Chem. Phys. 14(6), 2065 (2012)

211. S. Khanna and J. Lambe, Inelastic electron tunneling spectroscopy, Science 220(4604), 1345 (1983)

212. Y. Zhang, V. W. Brar, F. Wang, C. Girit, Y. Yayon, M. Panlasigui, A. Zettl, and M. F. Crommie, Giant phononinduced conductance in scanning tunnelling spectroscopy of gate-tunable graphene, Nat. Phys. 4(8), 627 (2008)

213. M. Ternes, Spin excitations and correlations in scanning tunneling spectroscopy, New J. Phys. 17(6), 063016 (2015)

214. S. Mishra, D. Beyer, K. Eimre, S. Kezilebieke, R. Berger, O. Gröning, C. A. Pignedoli, K. Müllen, P. Liljeroth, P. Ruffieux, X. Feng, and R. Fasel, Topological frustration induces unconventional magnetism in a nanographene, Nat. Nanotechnol. 15(1), 22 (2020) 
215. J. Su, W. Fan, P. Mutombo, X. Peng, S. Song, M. Ondráček, P. Golub, J. Brabec, L. Veis, M. Telychko, P. Jelínek, J. Wu, and J. Lu, On-surface synthesis and characterization of [7]triangulene quantum ring, Nano Lett. 21(1), $861(2021)$
216. J. Li, S. Sanz, M. Corso, D. J. Choi, D. Peña, T. Frederiksen, and J. I. Pascual, Single spin localization and manipulation in graphene open-shell nanostructures, Nat. Commun. 10, 200 (2019) 